\title{
Mitochondrial biogenesis is required for the anchorage- independent survival and propagation of stem-like cancer cells
}

\author{
Arianna De Luca ${ }^{1,2,3, *}$, Marco Fiorillo ${ }^{1,2,3, *}$, Maria Peiris-Pagès ${ }^{1,2}$, Bela Ozsvari ${ }^{1,2}$, \\ Duncan L. Smith ${ }^{4}$, Rosa Sanchez-Alvarez ${ }^{1,2}$, Ubaldo E. Martinez-Outschoorn ${ }^{5}$, Anna \\ Rita Cappello ${ }^{3}$, Vincenzo Pezzi ${ }^{3}$, Michael P. Lisanti ${ }^{1,2}$ and Federica Sotgia ${ }^{1,2}$ \\ ${ }^{1}$ The Breakthrough Breast Cancer Research Unit, Institute of Cancer Sciences, University of Manchester, UK \\ 2 The Manchester Centre for Cellular Metabolism (MCCM), Institute of Cancer Sciences, University of Manchester, UK \\ ${ }^{3}$ Department of Pharmacy, Health and Nutritional Sciences, University of Calabria, Arcavacata di Rende (CS), Italy \\ ${ }^{4}$ The Cancer Research UK Manchester Institute, University of Manchester, UK \\ ${ }^{5}$ The Sidney Kimmel Cancer Center, Philadelphia, PA, USA \\ * These authors contributed equally and should be considered co-first authors \\ Correspondence to: Michael P. Lisanti, email: michaelp.lisanti@gmail.com \\ Federica Sotgia, email: fsotgia@gmail.com \\ Keywords: XCT790, doxycycline, drug repurposing, ERR-a, PGC1-a/ $\beta$ \\ Abbreviations: CSCs, cancer stem-like cells; TICs, tumor-initiating stem-like cells. \\ Received: February 05, $2015 \quad$ Accepted: May 30, $2015 \quad$ Published: June 09, 2015
}

This is an open-access article distributed under the terms of the Creative Commons Attribution License, which permits unrestricted use, distribution, and reproduction in any medium, provided the original author and source are credited.

\section{ABSTRACT}

Here, we show that new mitochondrial biogenesis is required for the anchorage independent survival and propagation of cancer stem-like cells (CSCs). More specifically, we used the drug XCT790 as an investigational tool, as it functions as a specific inhibitor of the ERRa-PGC1 signaling pathway, which governs mitochondrial biogenesis. Interestingly, our results directly demonstrate that XCT790 efficiently blocks both the survival and propagation of tumor initiating stem-like cells (TICs), using the MCF7 cell line as a model system. Mechanistically, we show that XCT790 suppresses the activity of several independent signaling pathways that are normally required for the survival of CSCs, such as Sonic hedgehog, TGFB-SMAD, STAT3, and Wnt signaling. We also show that XCT790 markedly reduces oxidative mitochondrial metabolism (OXPHOS) and that XCT790-mediated inhibition of CSC propagation can be prevented or reversed by Acetyl-L-Carnitine (ALCAR), a mitochondrial fuel. Consistent with our findings, over-expression of ERRa significantly enhances the efficiency of mammosphere formation, which can be blocked by treatment with mitochondrial inhibitors. Similarly, mammosphere formation augmented by FOXM1, a downstream target of Wnt/ $\beta$-catenin signaling, can also be blocked by treatment with three different classes of mitochondrial inhibitors (XCT790, oligomycin A, or doxycycline). In this context, our unbiased proteomics analysis reveals that FOXM1 drives the expression of $>90$ protein targets associated with mitochondrial biogenesis, glycolysis, the EMT and protein synthesis in MCF7 cells, processes which are characteristic of an anabolic CSC phenotype. Finally, doxycycline is an FDA-approved antibiotic, which is very well-tolerated in patients. As such, doxycycline could be re-purposed clinically as a 'safe' mitochondrial inhibitor, to target FOXM1 and mitochondrial biogenesis in CSCs, to prevent tumor recurrence and distant metastasis, thereby avoiding patient relapse. 


\section{INTRODUCTION}

Tumor-initiating stem-like cells (TICs) are a small sub-population of tumor cells that are resistant to most anti-cancer therapies, including radio- and chemotherapy, and are able to expand and regenerate tumors, after conventional therapy is completed [1-4]. As such, TICs are responsible for tumor recurrence, metastatic dissemination, and, ultimately, patient death. As a consequence, TICs have become very attractive targets for novel cancer therapies. TICs share some features with stem cells, and many studies have investigated the signaling pathways regulating their proliferation, asymmetric cell division and migrating properties, as well as their ability to undergo proliferation under anchorage-independent conditions [57]. In fact, this latter property is being widely exploited to isolate TICs, which are able to survive and clonally expand as tumor-spheres, when placed in non-adherent settings [8]. Tumor-spheres generated from breast cancer cells are known as mammospheres.

The metabolic requirements of TICs remain a fairly unexplored area of investigation. We and others have recently shown that TICs rely mainly on mitochondrial metabolism, as compared to the more differentiated tumor cell population [9-12]. Also, it was previously shown that the radio-resistance of TICs from gliomas [13] and breast cancers [14] correlates with higher mitochondrial respiration.

We have demonstrated that $>60$ mitochondrial proteins are up-regulated in mammospheres derived from breast cancer cell lines (MCF7 and T47D), relative to cells grown in monolayers. Furthermore, pharmacological inhibition with oligomycin A, an inhibitor of the ATP synthase, greatly reduced mammosphere formation [11]. More importantly, treatment with doxycycline, an FDAapproved antibiotic targeting mitochondrial ribosomes as a known 'side effect', inhibited the formation of tumorspheres generated from cell lines across several different tumor types, as well as from primary tumor samples [12].

These studies indicate that mitochondria in TICs are potentially druggable targets for the prevention of tumor recurrence and metastasis. The aim of our current study was to further validate mitochondrial function as a critical target for the development of new anti-cancer therapies. Here, we employed XCT790 as an investigational compound to inhibit mitochondrial oxidative phosphorylation [15]. XCT790 is a well-established inverse agonist of Estrogen-Related Receptor $\alpha$ (ERR $\alpha)$, which functions as an essential cofactor of PGC1 $\alpha$. Importantly, $\mathrm{PGC} 1 \alpha$ is required for the transcription of nuclear-encoded mitochondrial genes and drives mitochondrial biogenesis [16, 17]. XCT790 has been shown to inhibit colony formation in soft agar induced by PGC1 $\alpha$ [18], and to induce cell death in chemo-resistant cancer cells [19]. However, to our knowledge, no studies have previously investigated the effects of XCT790 on the propagation and survival of TICs.

Here, our results show that XCT790 inhibits the proliferation of TICs from MCF7 breast cancer cells, as assessed using i) mammosphere formation and by ii) CD44/CD24 immuno-staining, coupled with FACS analysis. We also demonstrate that XCT790 suppresses the activation of well-established pathways governing TIC proliferation and survival, including Sonic hedgehog, TGF $\beta$-SMAD, STAT3, and Wnt signaling. As expected, XCT790 profoundly reduced mitochondrial respiration. Importantly, XCT790-induced TIC inhibition could be rescued by treatment with Acetyl-L-Carnitine (ALCAR), a mitochondrial fuel and cofactor, which enhances mitochondrial oxidative metabolism and biogenesis [20]. We also show that overexpression of ERR $\alpha$ is sufficient to augment MCF7 cell mammosphere formation, which can be blocked by treatment with mitochondrial inhibitors, such as XCT790 and oligomycin A, an inhibitor of ATP synthase. Finally, we show that mammosphere formation driven by FOXM1, a transcription factor downstream of $\mathrm{Wnt} / \beta$-catening signaling that governs stemness [21-24], can also be blocked by treatment with three different classes of mitochondrial inhibitors (XCT790, oligomycin A, or doxycycline).

Thus, our results firmly establish that mitochondrial biogenesis and metabolism are required for the survival of TICs, indicating that mitochondrial-targeted therapies are a promising novel strategy for targeting TICs for the treatment of relapsed and resistant cancers. Mitochondrialtargeted therapies should also be considered for cancer prevention.

\section{RESULTS}

\section{Mammosphere formation in MCF7 cells depends on mitochondrial function}

We have previously shown that mitochondrial proteins are up-regulated in mammospheres derived from breast cancer cell lines (MCF7 and T47D), relative to cells grown in monolayers [11]. Furthermore, pharmacological inhibition with oligomycin A, an inhibitor of ATP synthase, greatly reduced mammosphere formation [11]. More importantly, treatment with doxycycline, an FDAapproved antibiotic that targets mitochondrial ribosomes as a known 'side effect', inhibited the formation of tumorspheres derived from 12 cell lines across eight different tumor types, as well as from primary tumor samples [12].

To further investigate the role of mitochondria in TICs, here we employed a well-established inverse agonist of ERR $\alpha$, namely XCT790. ERR $\alpha$ is a cofactor of $\mathrm{PGC} 1 \alpha / \beta$, which is required for the transcription of nuclear mitochondrial genes and mitochondrial biogenesis $[16,17]$. As such, XCT790 is believed to 
suppress mitochondrial function. Notably, treatment with XCT790 dose-dependently inhibits MCF7 mammosphere formation, with an IC-50 of $10 \mu \mathrm{M}$ (Figure 1A). We also validated that XCT790 dose-dependently reduces the protein expression of PGC1 $\alpha$ in MCF7 cell monolayers by immuno-blot analysis (data not shown), by up to 70 $80 \%$, over this concentration range.

These results were independently confirmed by evaluating the expression of bona-fide TIC markers CD44/ CD24 by FACS. Under these conditions, CD44(+)high/ CD24(-)low cells are considered to represent the TIC sub-population. XCT790 treatment significantly reduced the number of CD44(+)high/CD24(-)low cells in a dosedependent fashion, relative to vehicle alone controls (Figure 1B).

We next set out to investigate if XCT790 can target TICs in the presence of the total cancer cell population. To this end, MCF7 cells were treated with XCT790 (at 5 or 10 $\mu \mathrm{M})$ as monolayers for 2 days and then re-plated on lowattachment plates in the absence of XCT790, to generate mammospheres for 5 days. Under these conditions, XCT790 pre-treatment dose-dependently reduced MCF7 cell mammosphere formation, by up to $\sim 70 \%$ (Figure $1 \mathrm{C}$ ), indicating that XCT790 can target the TIC population also

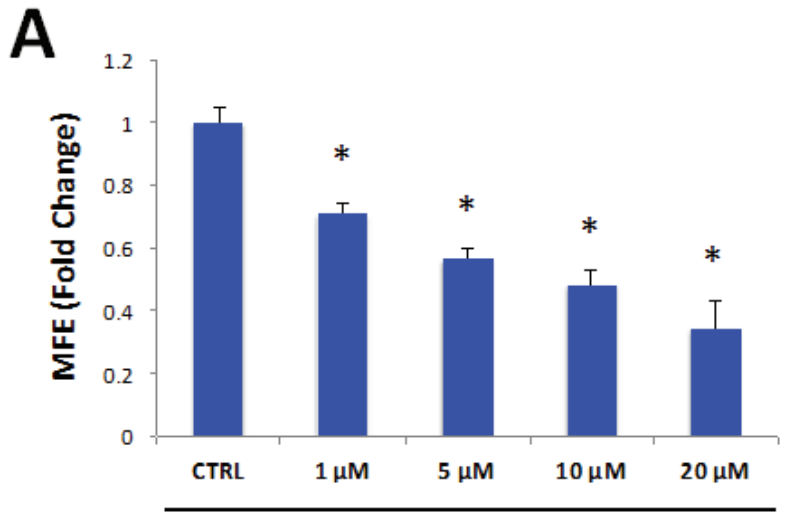

XCT790
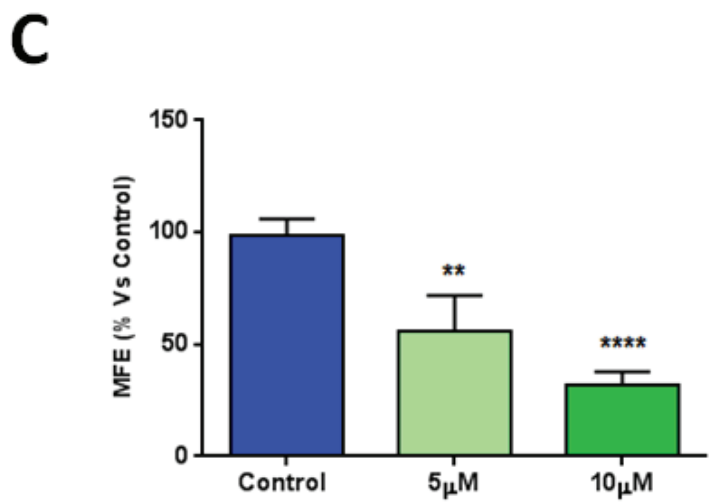

B

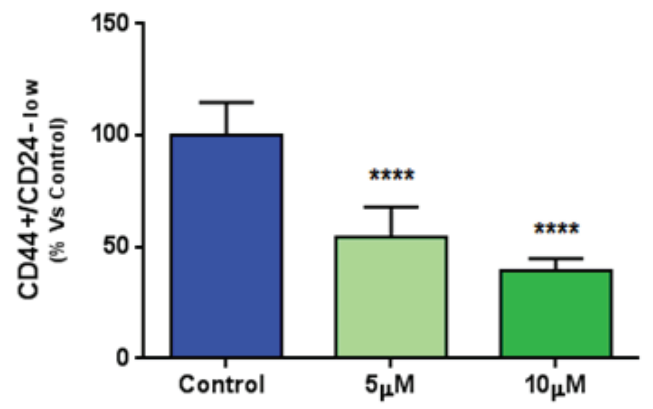

D

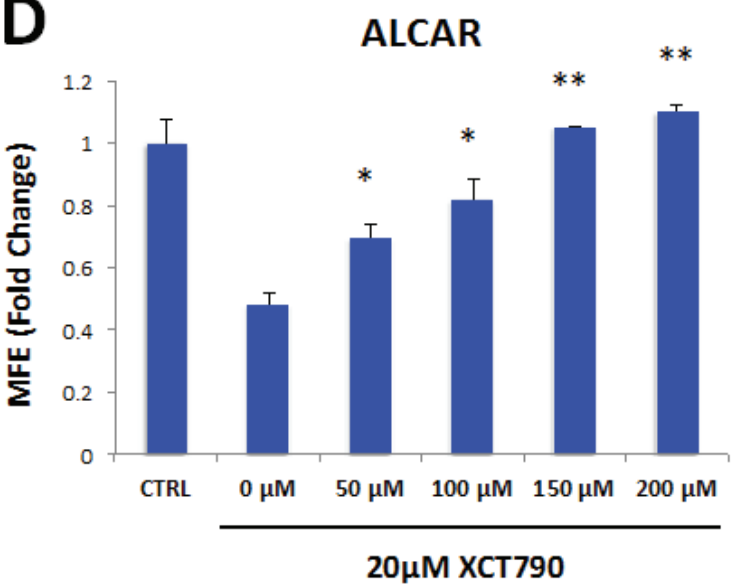

Figure 1: MCF7 cell 3D-spheroid formation depends on mitochondrial function. A. The well-established ERR $\alpha$ inverse agonist, namely XCT790, dose-dependently inhibits MCF7 mammosphere formation, with an IC-50 of $10 \mu \mathrm{M}$. ERR $\alpha$ is a cofactor of PGC1 $\alpha$, which is required for the transcription of mitochondrial genes and mitochondrial biogenesis. * $p<0.01$ evaluated by Student's $t$ test. B. MCF7 cells were pre-treated with XCT790 (at 5 or $10 \mu \mathrm{M}$ ) as monolayers for 2 days and then re-plated on low-attachment plates in the absence of XCT790, for anoikis assay for 10 hours. Expression of TIC markers CD24 and CD44 was analyzed by FACS. Note that XCT790 pre-treatment dose-dependently reduced the number of $\mathrm{CD} 44(+)$ high/CD24(-)low cells, which are considered the TICs population. $* * * * p$ $<0.00001$ evaluated with one-way ANOVA. C. MCF7 cells were pre-treated with XCT790 (at 5 or $10 \mu \mathrm{M}$ ) as monolayers for 2 days and then re-plated on low-attachment plates in the absence of XCT790, for mammosphere assay for 5 days. Under these conditions, XCT790 pre-treatment dose-dependently reduced MCF7 cell mammosphere formation, by up to $\sim 70 \%$. ** $p<0.001$ and $* * * * p<0.00001$ evaluated with one-way ANOVA. D. Treatment with the mitochondrial cofactor Acetyl L-Carnitine (ALCAR) rescues the decreased mammosphere formation induced by XCT790. Mammosphere formation was assessed upon treatment with $20 \mu \mathrm{M} \mathrm{XCT790} \mathrm{and} \mathrm{increasing} \mathrm{concentration}$ of ALCAR. Thus, mitochondrial function is required for the efficient clonal expansion and anchorage-independent growth of TICs. ${ }^{*} p$ $<0.05 ; * *<<0.001$, relative to XCT790 only treated cells ( $0 \mu \mathrm{M}$ ALCAR) evaluated by Student's $t$ test. MFE: mammosphere forming efficiency. 
when present in a heterogeneous cell population.

Also, we asked if decreased mammosphere formation induced by XCT790 could be rescued by treatment with the mitochondrial cofactor AcetylL-Carnitine (ALCAR). ALCAR plays a key role in mitochondrial oxidative metabolism, by enhancing fatty acid $\beta$-oxidation [20]. ALCAR stimulates mitochondrial biogenesis and is also directly converted to acetyl-CoA, a mitochondrial fuel [20]. To this end, mammosphere formation was assessed after treatment with XCT790 (at $20 \mu \mathrm{M}$ ) and increasing concentrations of ALCAR. Figure 1D shows that ALCAR rescues the decrease in mammosphere formation induced by XCT790, in a dose-dependent manner. Thus, mitochondrial function is required for the efficient clonal expansion and anchorageindependent growth of TICs.

We then examined if XCT790 affects the viability of the total cancer cell population, or if it specifically inhibits the viability of MCF7 cells in mammospheres. To this end, MCF7 cells were treated with increasing concentrations of XCT790 as monolayers for 3 days (Figure 2A) or 5 days (Figure 2B). Cell viability was then assessed using the SRB assay. Note that 5-day treatment did not affect the viability of the MCF7 cell monolayers, as profoundly as MCF7 cell mammospheres (Figure 2B). For example, treatment with $10 \mu \mathrm{M}$ XCT790 reduces mammosphere formation by $50 \%$ (Figure 1A), whereas the viability of monolayer cells is reduced by only $20 \%$. Thus, XCT790 preferentially reduces the viability of MCF7 cell mammospheres, relative to bulk cancer cells.

A

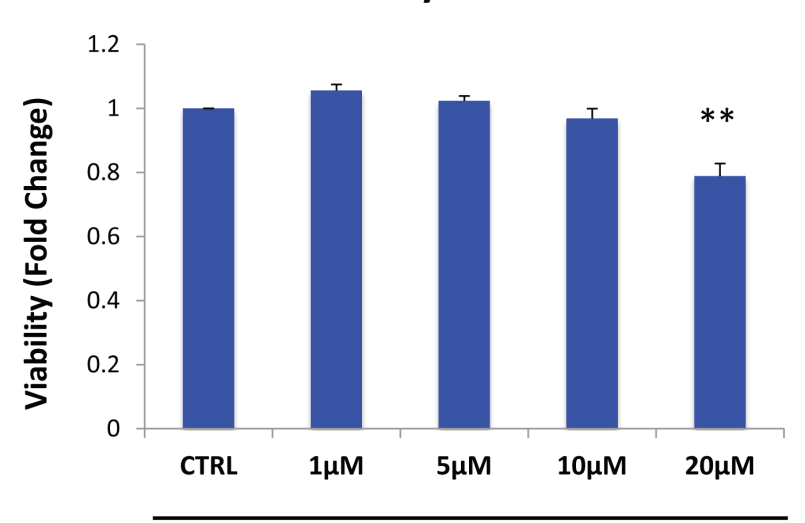

XCT790

\section{XCT790 inhibits the activation of several stem cell} related signaling pathways

To further corroborate the idea that XCT790 inhibits cancer stem cell-like features, we next analyzed the effects of XCT790 on a series of well-established signaling pathways, which have been shown to promote stemness. For this purpose, we employed a panel of eight MCF7 cell lines carrying different luciferase reporters [25], to monitor the activation state of a variety of different signaling networks, including Sonic hedgehog, TGF $\beta$-SMAD, STAT3, Wnt, Interferon (IFN)- $\alpha / \beta-$ STAT1/2, NRF2dependent antioxidant responses, IFN- $\gamma$-STAT1 and Notch pathways. Notably, several pathways were significantly inhibited by XCT790 treatment, including stem cell signaling (Sonic hedgehog, TGF $\beta$-SMAD, STAT3, Wnt) and IFN- $\alpha / \beta-$ STAT1/2 signaling (Figure 3A). However, no effects were observed on the NRF2-antioxidant response, IFN- $\gamma$-STAT1 and the Notch pathways (Figure 3B). Thus, XCT790 inhibits the activation of several signal transduction pathways related to cancer stem-like features.

\section{XCT790 inhibits mitochondrial respiration}

Next, we set out to investigate the mechanism(s) by which XCT790 inhibits mammosphere formation and CSC features. XCT790 is an inverse agonist of ERR $\alpha$, which is a cofactor of $\mathrm{PGC} 1 \alpha$ required for the transcription of mitochondrial genes and mitochondrial biogenesis. Thus, the metabolic profile of MCF7 cells treated with XCT790 was analyzed. To this end, MCF7 cells were treated with XCT790 for 48 hours, stained with various MitoTracker

B

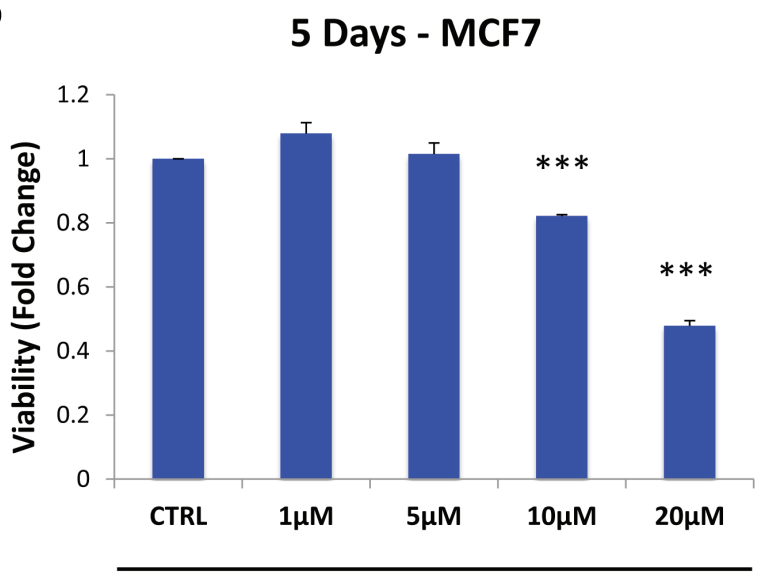

XCT790

Figure 2: XCT790 preferentially reduces the viability of MCF7 cells in mammospheres, relative to bulk cancer cells. MCF7 cells were treated with XCT790 $(1,5,10,20 \mu \mathrm{M})$ as monolayers for 3 days A. or 5 days B. Cell viability was assessed by SRB assay. Note that 5-day treatment did not affect the viability of the MCF7 cell monolayers as profoundly as MCF7 cell mammospheres. For example, treatment with $10 \mu \mathrm{M}$ XCT790 reduces mammosphere formation by $50 \%$ (Figure 1A), whereas viability of monolayer cells is reduced by $20 \%$. ** $p<0.01 ; * * p<0.001$ evaluated by Student's $t$ test. 

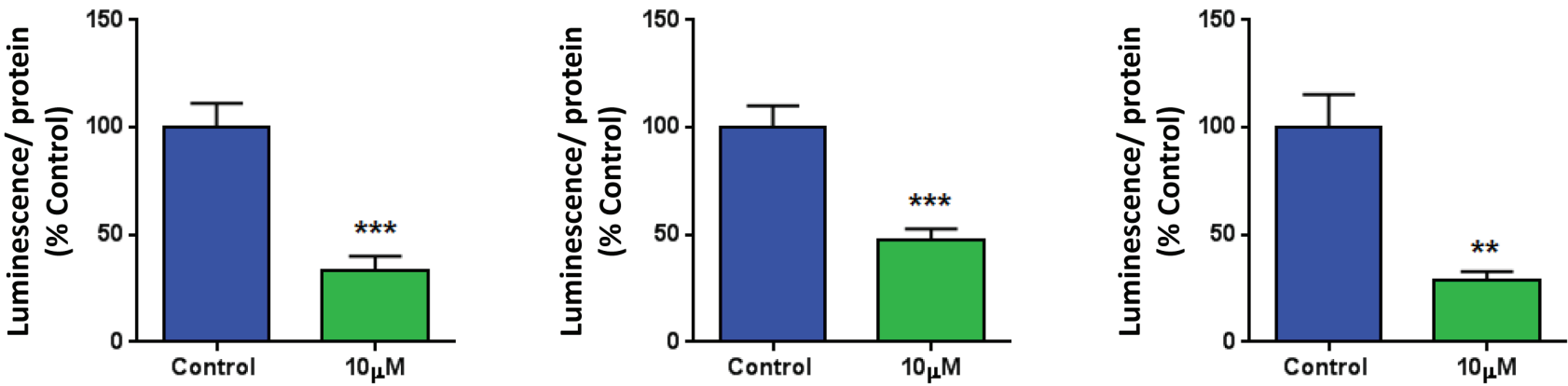

Wnt-Signaling

IFN $\alpha / \beta$-(Stat1/2)-Signaling
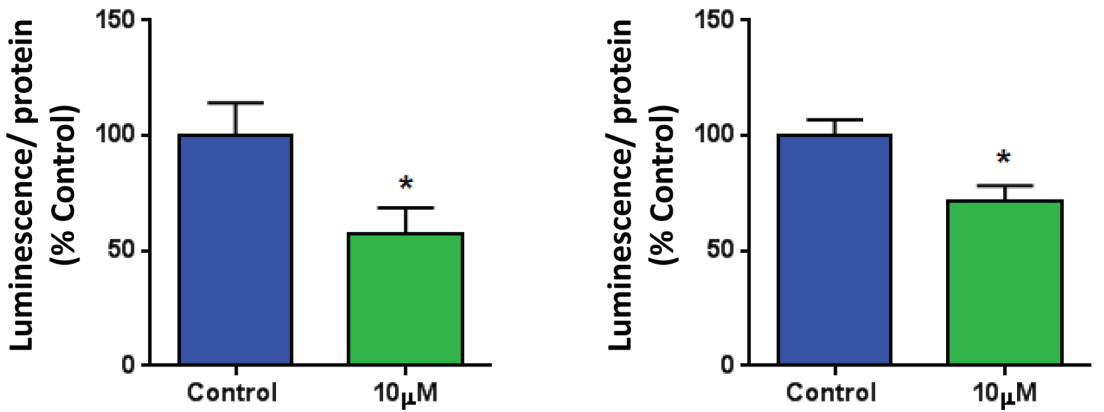

B

ARE/Nrf2-Antioxidant

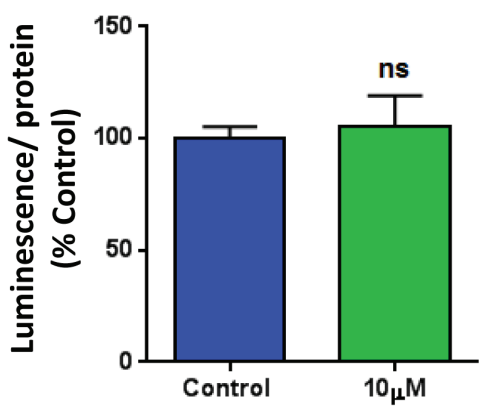

INFY-STAT1-Signaling

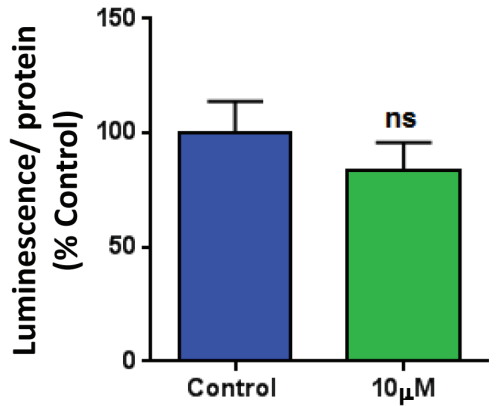

Notch-Signaling

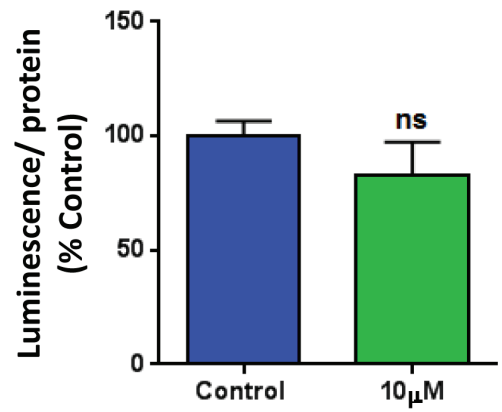

Figure 3: XCT790 inhibits signaling pathways related to cancer stem cells, and interferon. MCF7 breast cancer cells carrying luciferase-reporters (Cignal, QIAGEN) were generated to monitor the activation of a variety of signaling networks, including Sonic hedgehog TGF $\beta$-SMAD, STAT3, Wnt, Interferon (IFN)- $\alpha / \beta$-STAT1/2, NRF2-dependent antioxidant responses, IFN- $\gamma$-STAT1 and Notch pathways. MCF7-Luc reporter cells were treated with XCT790 for 48 hours and luminescence was determined as a measure of pathway activation status. Luminescence was normalized by protein content. A. Note that XCT790 inhibits cancer stem cell signaling (Sonic hedgehog TGF $\beta$-SMAD, STAT3, Wnt), as well as IFN- $\alpha / \beta-S T A T 1 / 2$ signaling. B. No effects were observed for the NRF2-antioxidant responses, IFN- $\gamma$-STAT1 and Notch pathways. ${ }^{*} p<0.01 ; * p<0.001 ; * * *<0.0001$, using Student's t test. 
probes and analyzed by FACS. Figure 4A shows that XCT790 induces a decrease in mitochondrial membrane potential, as assessed with MitoTracker Orange, which accumulates in mitochondria with an active mitochondrial potential. Surprisingly, XCT790 induces also an increase in mitochondrial mass, as assessed with MitoTracker Green (Figure 4B), localizing to mitochondria regardless of mitochondrial membrane potential. Similar results were obtained with MitoTracker Deep Red, which also measures mitochondria mass (data not shown). Analysis of the ratio of mitochondrial membrane potential versus mitochondrial mass indicates that XCT790 induces a profound decrease in mitochondrial membrane potential per mitochondria (Figure 4C).

Also, the metabolic profile of MCF7 cells treated with XCT790 was examined using the Seahorse XFe96 analyzer, by employing a mitochondrial stress test. Notably, the oxygen consumption rate (OCR) was greatly reduced by treatment with XCT790 (Figure 5A). Further quantification revealed significant reductions in basal and maximal respiration (Figure 5B-5C), as well as ATP levels (Figure 5D), upon XCT790 treatment. Thus, XCT790 significantly reduces the rates of oxidative mitochondrial metabolism. To further validate these results, a glycolytic stress test was performed using the Seahorse XFe96 analyzer, by measuring the extracellular acidification rate (ECAR) of MCF7 cells treated with XCT790. Notably, XCT790 also significantly reduces ECAR, a marker of glycolysis (Figure 6A). Finally, plotting the ratio of OCR to ECAR demonstrates that XCT790 shifts MCF7 cells from a highly energetic to a metabolically quiescent state (Figure 6B).
ERR $\alpha$ expression is both necessary and sufficient to increase mammosphere formation in MCF7 cells

XCT790 is an inverse agonist of ERR $\alpha$. As XCT790 inhibits mammosphere formation, we then asked if ERR $\alpha$ expression is sufficient to promote $3 \mathrm{D}$-spheroid formation in MCF7 cells. To this end, ERR $\alpha$ was over-expressed in MCF7 cells using a lentiviral approach. Empty Vector (EV) cells were generated in parallel. Figure 7A shows that MCF7 cells over-expressing ERR $\alpha$ show a 50\% increase in mammosphere forming capacity. Notably, MCF7 cells over-expressing ERR $\alpha$ show increased mitochondrial membrane potential, as assessed by MitoTracker Orange, as well as increased mitochondrial mass, as assessed by MitoTracker Green and MitoTracker Deep Red, as expected (Figure 7B).

We then used a pharmacological approach to inhibit $\mathrm{ERR} \alpha$-driven mammosphere formation, using oligomycin A, a mitochondrial inhibitor, as well as XCT790, a specific $\mathrm{ERR} \alpha$ inverse agonist. Importantly, mammosphere formation induced by ERR $\alpha$ expression was inhibited by either treatment with oligomycin A (Figure 7C), or XCT790 (Figure 7D). These results indicate that ERR $\alpha$ activity and mitochondrial function are normally required for the clonal expansion of TICs.

\section{Mammosphere formation driven by FOXM1 requires mitochondrial function}

To examine if mitochondrial function is essential for TIC expansion driven by well-established stemlike signaling, we decided to overexpress FOXM1 in
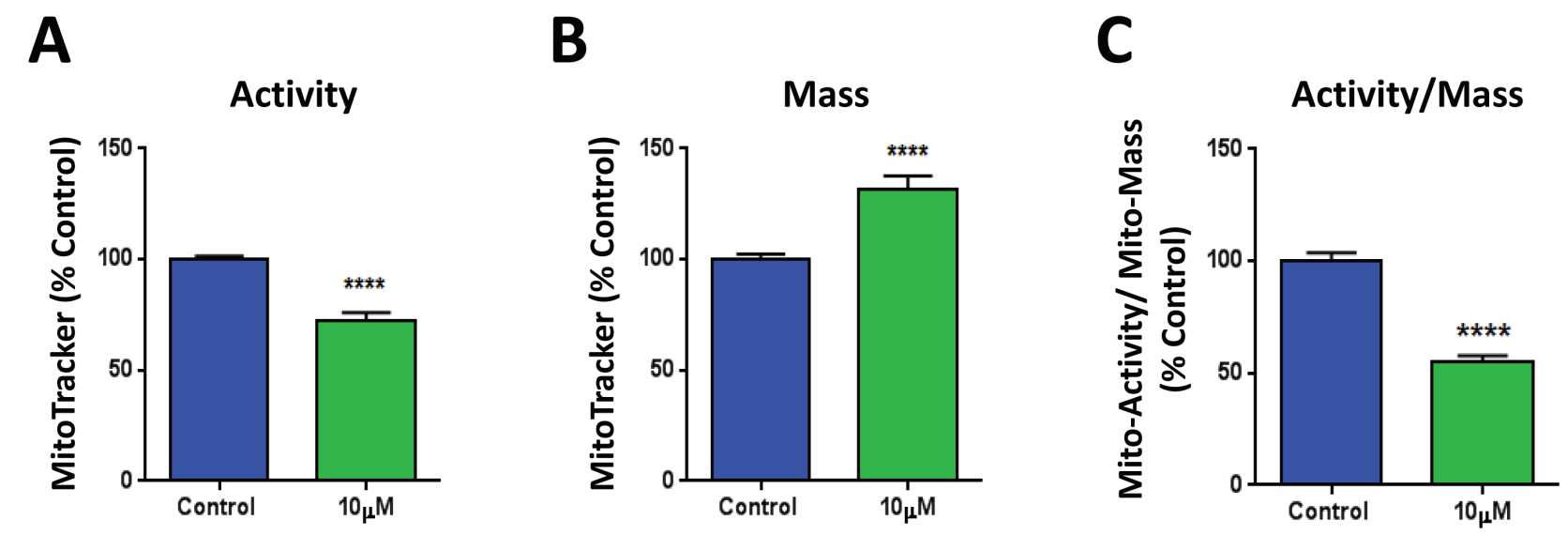

Figure 4: XCT790 induces a decrease in mitochondrial membrane potential, with an unexpected increase in mitochondrial mass. MCF7 cells were treated with XCT790 $(10 \mu \mathrm{M})$ as monolayers for 48 hours, stained with various MitoTracker probes and analyzed by FACS. A. XCT790 induces a decrease in mitochondrial membrane potential, as assessed with MitoTracker Orange, which accumulates in mitochondria with an active mitochondrial potential. B. XCT790 induces an unexpected increase in mitochondrial mass, as assessed with MitoTracker Green, which localizes to mitochondria regardless of mitochondrial membrane potential. C. Ratio of mitochondrial membrane potential (MitoTracker Orange), versus mitochondrial mass (MitoTracker Green) demonstrates that XCT790 induces a large decrease in mitochondrial membrane potential per mitochondria. ${ }^{* * * *} p<0.00001$ evaluated with Student's $t$ test. 

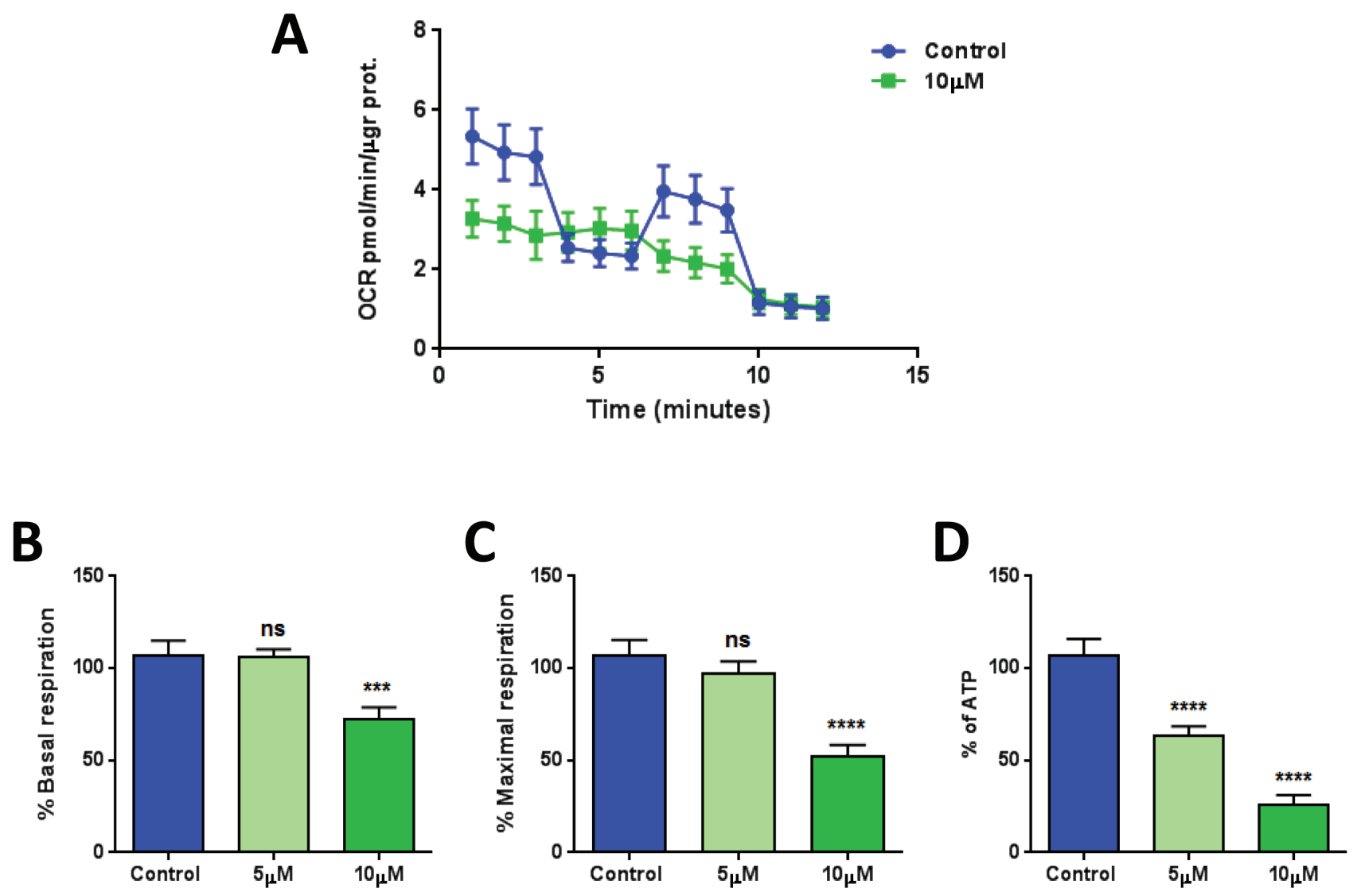

Figure 5: XCT790 quantitatively reduces mitochondrial respiration. The metabolic profile of MCF7 cell monolayers treated with XCT790 (5 or $10 \mu \mathrm{M})$ for 2-days was examined using the Seahorse XFe96 analyzer. A. Oxygen consumption rate (OCR) is significantly reduced by treatment with $10 \mu \mathrm{M}$ XCT790. B., C., D. Significant reductions in respiration (basal and maximal) and in ATP levels were observed upon XCT790 treatment. Thus, the rates of oxidative mitochondrial metabolism were significantly reduced by XCT790 treatment. $* * * p<0.0001, * * * * p<0.00001$ evaluated with one-way ANOVA.



B


Figure 6: XCT790 shifts MCF7 cells from a highly energetic to a metabolically quiescent state. A. Extracellular acidification rate (ECAR) of MCF7 cell monolayers treated with XCT790 $(10 \mu \mathrm{M})$ for 2-days was assessed using the Seahorse XFe96 analyzer. Note that XCT790 significantly reduces ECAR, a marker of glycolysis. ${ }^{*} p<0.01$ evaluated with Student's $t$ test. B. OCR plotted against ECAR. Note that MCF7 cells treated with XCT790 were shifted towards a more metabolically quiescent state. 


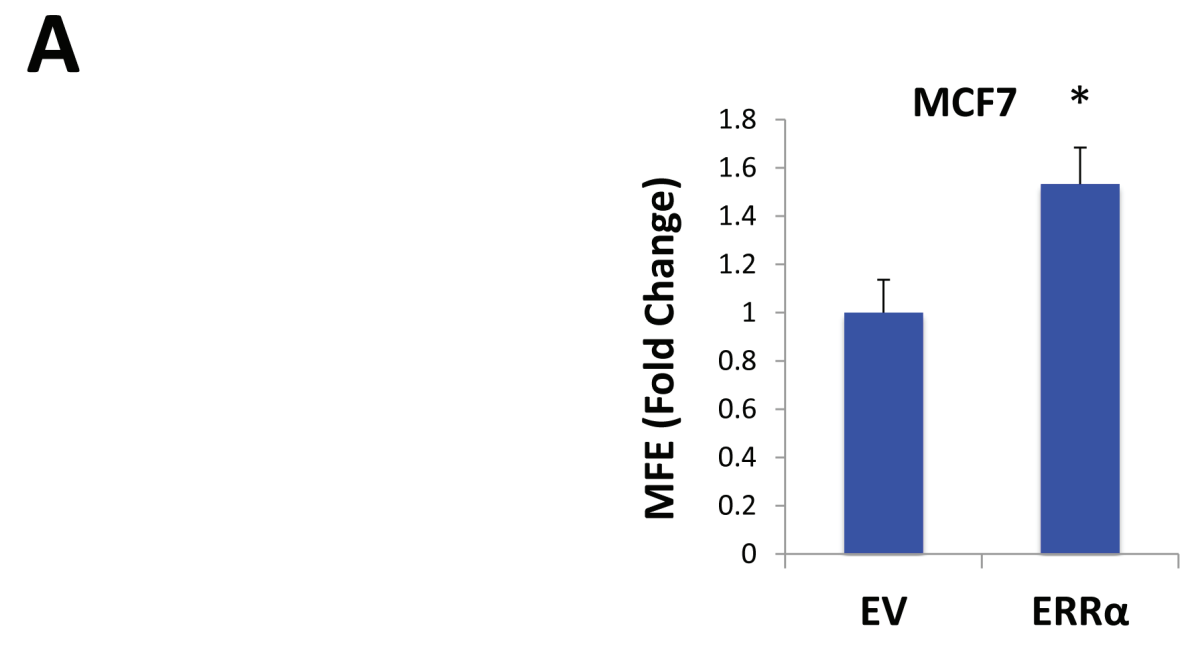

B

MitoTracker Orange

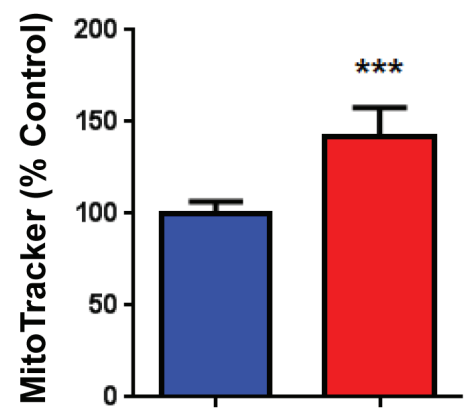

EV ERRa

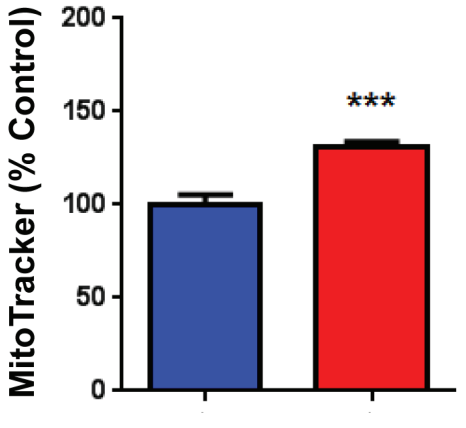

EV
ERRa
MitoTracker Deep Red

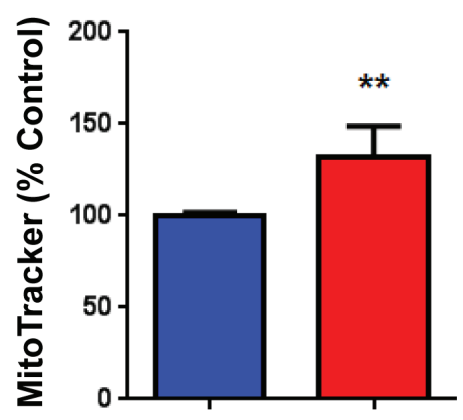

EV

ERRa

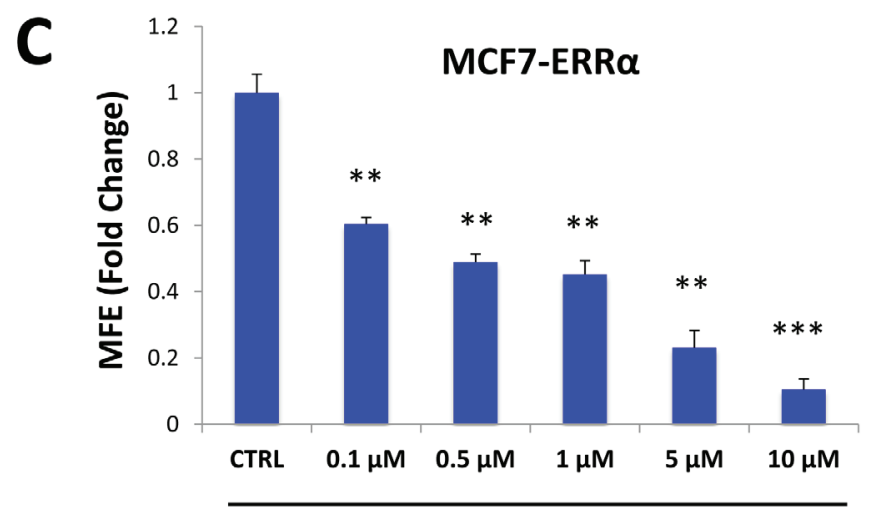

Oligomycin

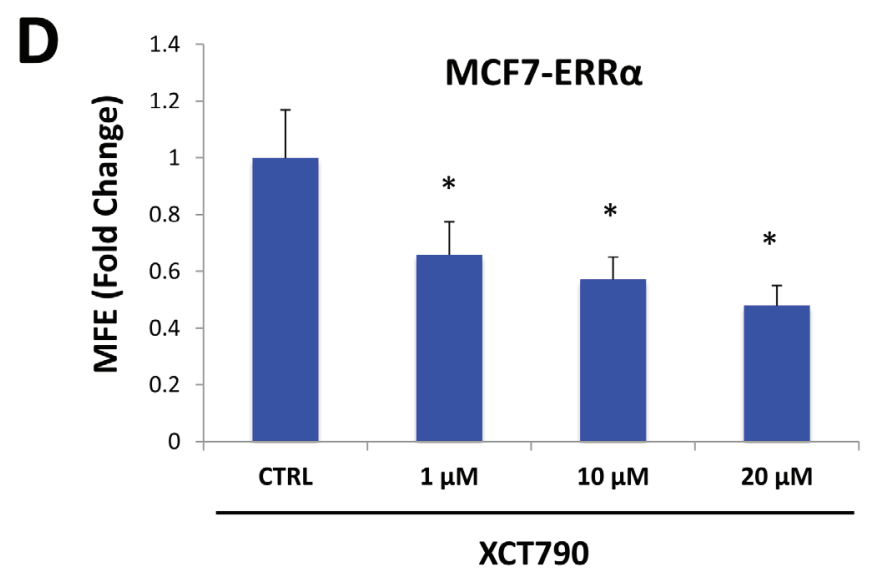

Figure 7: ERR $\alpha$ expression is required for 3D-spheroid formation in MCF7 cells. ERR $\alpha$ was over-expressed in MCF7 cells using a lentiviral approach. Empty Vector (EV) cells were generated in parallel. A. MCF7 cells overexpressing ERR $\alpha$ show a $50 \%$ increase in mammosphere forming capacity, relative to EV controls. B. MCF7 cells over-expressing ERR $\alpha$ show an increase of mitochondrial membrane potential, as assessed by MitoTracker Orange staining, as well as an increase in mitochondrial mass, as assessed by MitoTracker Green and MitoTracker Deep Red staining, as expected. C. Oligomycin A, an inhibitor of mitochondrial ATP synthase, inhibits mammosphere formation in MCF7 cells overexpressing ERR $\alpha$, indicating that mitochondrial function is required for ERR $\alpha$-driven mammosphere formation. D. XCT790, an ERR $\alpha$ inverse agonist, inhibits mammosphere formation in MCF7 cells over-expressing ERR $\alpha$. Thus, ERR $\alpha$ activity is required for the efficient clonal expansion of TICs. EV: Empty Vector. ${ }^{*} p<0.05,{ }^{* *} p<0.01,{ }^{* * *} p<0.001$ evaluated by Student's $t$ test. 
Table 1: MCF7-FOXM1 Cells Over-express Key Protein Molecules Functionally Related to Mitochondria, Glycolysis and the EMT.

Symbol Description

Fold-Upregulation

ANOVA

Mitochondrial-related proteins (27)

HSPD1 HSP60, $60 \mathrm{kDa}$ heat shock protein, mitochondrial

ACADVL Acyl-Coenzyme A dehydrogenase, very long chain, mitochondrial

ATP5B ATP synthase subunit beta, mitochondrial

COX4I1 Cytochrome c oxidase subunit 4 isoform 1, mitochondrial

SLC25A4 Solute carrier family 25 (ADP/ATP translocase), member 4, mitochondrial

PRKDC DNA-dependent protein kinase, catalytic (maintains mt-DNA copy number)

ABAT

4-aminobutyrate aminotransferase, mitochondria

PMPCA Mitochondrial-processing peptidase alpha subunit

CHCHD3 MICOS complex subunit MIC19, mitochondrial

NDUFS1 Mitochondrial NADH-ubiquinone oxidoreductase $75 \mathrm{kDa}$ subunit

HSPA9 Heat shock $70 \mathrm{kDa}$ protein 9 (Mortalin), mitochondrial

SUCLG2 Succinate-CoA ligase, GDP-forming, beta subunit

SLC25A3 Solute carrier family 25 (mitochondrial phosphate carrier), member 3

MTHFD2 methylene tetrahydrofolate dehydrogenase (NAD+dependent), mitochondrial

$\mathrm{MDH} 2 \quad$ Malate dehydrogenase 2, NAD (mitochondrial)

COX5A Cytochrome $c$ oxidase subunit 5A, mitochondrial

TIMM9 Translocase of inner mitochondrial membrane 9

IDH2 Isocitrate dehydrogenase [NADP], mitochondrial

PDHA1 Pyruvate dehydrogenase E1 component subunit alpha, mitochondrial

MRPL49 39S ribosomal protein L49, mitochondrial

ATP5A1 ATP synthase subunit alpha, mitochondrial

HADH2 Hydroxyacyl-Coenzyme A dehydrogenase, type II, mitochondrial (HSD17B10)

HADHB Trifunctional enzyme subunit beta, mitochondrial

ALDH18A1 Delta-1-pyrroline-5-carboxylate synthase, mitochondrial

GLRX5 Glutaredoxin-related protein 5, mitochondrial

PMPCB Mitochondrial-processing peptidase subunit beta

MRPS22 28S ribosomal protein S22, mitochondrial

12.35

12.14

10.74

9.44

5.97

2.43

2.28

2.21

2.04

1.99

1.90

1.88

1.84

1.83

1.79

1.78

1.78

1.70

1.67

1.63

1.60

1.55

1.55

1.55

1.97

1.69

1.51

10.02

8.35

7.72

5.49

5.44

2.81

1.86

1.81

1.74

1.62

23.94

10.85

8.11

6.37

5.74

4.87

4.70

4.70

4.40

4.32

3.84

3.47

3.12

2.44

2.38

2.35

2.29

2.11

2.05

2.04

2.01

1.93

1.84

1.82

1.81

1.80

1.68

1.65

1.59

1.54

1.52
0.02

0.001

0.0006

0.002

0.003

0.0005

0.0002

0.03

0.03

7.61E-05

0.049

0.01

0.037

0.046

0.03

0.0045

0.04

0.01

0.037

0.04

0.03

0.04

0.049

0.02

0.03

0.03

0.049

0.0003

0.003

$6.65 \mathrm{E}-05$

0.0002

$6.37 \mathrm{E}-05$

0.02

0.005

0.004

0.04

0.0001

7.17E-06

0.046

0.0002

9.67E-05

2.35E-05

4.54E-06

0.0005

0.006

0.003

0.05

0.0007

4.69E-05

0.001

0.007

0.01

0.002

0.02

0.03

0.03

0.03

2.01E-05

0.008

0.047

0.05

0.009

0.0006

0.04

0.04

0.02

0.04

0.048 
Table 2: MCF7-FOXM1 Cells Over-express Key Protein Molecules Functionally Related to Ribosomes and Protein Synthesis.

Symbol Description

Ribosome-related proteins (9)

RPL15 60S ribosomal protein L15

RPL8 $\quad 60 S$ ribosomal protein L8

RPS3A 40S ribosomal protein S3A

RPL7 $\quad 60 S$ ribosomal protein L7

RPL10A 60S ribosomal protein L10A

RPL7A $60 S$ ribosomal protein L7A

RRP1B Ribosomal RNA processing protein 1 homolog B

MRPL49 39S ribosomal protein L49, mitochondrial

MRPS22 28S ribosomal protein S22, mitochondrial

Translation initiation factors (4)

EIF4G3 Eukaryotic translation initiation factor 4 gamma 3

EIF3C Eukaryotic translation initiation factor 3 subunit $C$

EIF4B

Eukaryotic translation initiation factor $4 \mathrm{~B}$

EIF3S4 Eukaryotic translation initiation factor 3 subunit $G$

Elongation factors (5)

EEF1A1 Elongation factor 1-alpha 1

EEF1A2 Elongation factor 1-alpha 2

EEF1G Elongation factor 1-gamma

EFTUD2 Elongation factor Tu GTP binding domain containing 2

EEF1D Elongation factor 1-delta

Enzymes for tRNA synthesis (6)

GARS Glycine--tRNA ligase

DARS Aspartate--tRNA ligase, cytoplasmic

TARS Threonyl-tRNA synthetase, cytoplasmic

HARS Histidine--tRNA ligase, cytoplasmic

RARS Arginine--tRNA ligase, cytoplasmic

AARS Alanine--tRNA ligase, cytoplasmic

Protein folding chaperones (heat shock proteins) (8)

HSPD1 Heat shock 60kDa protein 1 (Chaperonin), mitochondrial

HSPB1 Heat shock protein beta-1

HSPA8 Heat shock cognate $71 \mathrm{kDa}$ protein

HSP90AB1 Heat shock protein HSP 90-beta

CLGN Calmegin (ER chaperone)

HSPA1A Heat shock $70 \mathrm{kDa}$ protein 1

HSPH 1 Heat shock $105 \mathrm{kDa} / 110 \mathrm{kDa}$ protein 1

HSPA6 Heat shock $70 \mathrm{kDa}$ protein 6

Fold-Upregulation

ANOVA

$\begin{array}{ll}5.49 & 0.003 \\ 4.41 & 0.01 \\ 1.78 & 0.046 \\ 1.71 & 0.02 \\ 1.69 & 0.006 \\ 1.68 & 0.02 \\ 1.66 & 0.05 \\ 1.63 & 0.04 \\ 1.51 & 0.049\end{array}$

5.72

2.44

1.83

1.55

3.19E-06

0.001

0.04

0.04

5.63

4.36

3.03

2.84

2.16

4.50

2.28

1.82

1.82

1.70

1.56

0.0004

0.05

0.003

4.94E-05

0.003

0.0006

0.01

0.01

0.03

0.01

0.03

$\begin{array}{ll}12.35 & 0.02 \\ 3.00 & 0.009 \\ 2.20 & 0.002 \\ 1.97 & 9.13 \mathrm{E}-07 \\ 1.59 & 0.026 \\ 1.57 & 0.002 \\ 1.55 & 0.02 \\ 1.55 & 0.006\end{array}$

MCF7 cells. Empty Vector (EV) control cells were generated in parallel. The transcription factor FOXM1 was recently shown to induce the expansion of human normal stem cells, as well as of cancer stem-like cells [21-24]. Mechanistically, it is believed that FOXM1 acts downstream of key signaling pathways essential for stem cell regulation and tumorigenesis, such as $\mathrm{Wnt} / \beta$-catenin and $14-3-3 \zeta$ signaling cells $[22,26]$.

MCF7 cells over-expressing FOXM1 show a 3.3fold increase in mammosphere formation, relative to empty-vector controls, as expected (Figure 8A). In order to better dissect the molecular changes driven by FOXM1, MCF7 cell monolayers over-expressing FOXM1 and EV control cells were subjected to proteomics analysis. Proteomic datasets of up-regulated proteins in the MCF7FOXM1 cells were then analyzed with respect to their metabolic protein profiles.

Table 1 shows that several key molecules related to mitochondria, glycolysis and the EMT are up-regulated in FOXM1-over-expressing MCF7 cells, indicating that FOXM1-induced stemness is associated with increased metabolic flexibility. Notably, keratin-19 (KRT19), a well-established marker of circulating tumor cells and CSCs, is increased by $>20$-fold in MCF7-FOXM1 cells. Also, four key mitochondrial proteins (HSPD1, ACADVL, ATP5B, COX4I1) are increased by $>10$-fold each in MCF7-FOXM1 cells; SLC25A4 (the mitochondrial ATP/ ADP exchanger) is increased by nearly 6 -fold. Similarly, twenty-two other mitochondrial proteins are increased by $>1.5$ fold.

Moreover, Table 2 shows that several key molecules related to protein synthesis are also up-regulated in FOXM1-overexpressing MCF7 cells. We have previously shown that protein synthesis is a mechanism for enhancing the proliferation of TICs, and that known inhibitors of protein synthesis, such as puromycin and rapamycin, are very effective at reducing mammosphere formation [27]. Similarly, the EMT is also regarded as a key feature of TICs $[28,29]$. Consistent with the notion that FOXM1 drives stemness, ribosome-related proteins and EMT markers are increased by FOXM1 expression (Tables 1 and 2). 
Table 3. FOXM1 Targets are Transcriptionally Up-regulated in Human Breast Cancer: Mitochondria, Glycolysis and the EMT.

\begin{tabular}{|c|c|c|c|}
\hline Symbol & Gene Description & $\begin{array}{l}\text { Up-regulation } \\
\text { (fold-change) }\end{array}$ & P-value \\
\hline \multicolumn{4}{|c|}{ Mitochondrial-related proteins (23) } \\
\hline ATP5B & ATP synthase subunit beta, mitochondrial & 5.04 & $2.75 \mathrm{E}-06$ \\
\hline ATP5A1 & ATP synthase subunit alpha, mitochondrial & 5.01 & 3.09E-06 \\
\hline MRPL49 & $39 S$ ribosomal protein L49, mitochondrial & 4.94 & 3.93E-06 \\
\hline $\mathrm{MDH} 2$ & Malate dehydrogenase 2, NAD (mitochondrial) & 4.18 & $5.32 \mathrm{E}-05$ \\
\hline GLRX5 & Glutaredoxin-related protein 5 , mitochondrial & 4.07 & 7.83E-05 \\
\hline PMPCB & Mitochondrial-processing peptidase subunit beta & 3.81 & 1.77E-04 \\
\hline SLC25A3 & Solute carrier family 25 (mitochondrial phosphate carrier), member 3 & 3.76 & 2.09E-04 \\
\hline HSPA9 & Heat shock 70kDa protein 9 (Mortalin), mitochondrial & 3.69 & $2.64 \mathrm{E}-04$ \\
\hline COX5A & Cytochrome $\mathrm{c}$ oxidase subunit $5 \mathrm{~A}$, mitochondrial & 3.62 & $3.22 \mathrm{E}-04$ \\
\hline TIMM9 & Translocase of inner mitochondrial membrane 9 & 3.58 & $3.69 \mathrm{E}-04$ \\
\hline HSPD1 & HSP60, $60 \mathrm{kDa}$ heat shock protein, mitochondrial & 3.42 & 5.93E-04 \\
\hline COX4I1 & Cytochrome $\mathrm{c}$ oxidase subunit 4 isoform 1 , mitochondrial & 3.39 & $6.61 \mathrm{E}-04$ \\
\hline MRPS22 & $28 \mathrm{~S}$ ribosomal protein $\mathrm{S} 22$, mitochondrial & 3.27 & $9.31 \mathrm{E}-04$ \\
\hline NDUFS1 & Mitochondrial NADH-ubiquinone oxidoreductase 75 kDa subunit & 3.20 & 1.15E-03 \\
\hline HADHB & Trifunctional enzyme subunit beta, mitochondrial & 3.06 & $1.73 \mathrm{E}-03$ \\
\hline SUCLG2 & Succinate-CoA ligase, GDP-forming, beta subunit & 3.03 & 1.89E-03 \\
\hline $\mathrm{CHCHD} 3$ & MICOS complex subunit MIC19, mitochondrial & 2.74 & 4.14E-03 \\
\hline MTHFD2 & methylene tetrahydrofolate dehydrogenase (NAD+dependent), mitochondrial & 2.67 & $4.98 \mathrm{E}-03$ \\
\hline $\mathrm{IDH} 2$ & Isocitrate dehydrogenase [NADP], mitochondrial & 2.46 & 8.55E-03 \\
\hline PRKDC & DNA-dependent protein kinase, catalytic (maintains mt-DNA copy number) & 2.14 & $1.85 \mathrm{E}-02$ \\
\hline ABAT & 4-aminobutyrate aminotransferase, mitochondrial & 2.08 & 2.14E-02 \\
\hline PDHA1 & Pyruvate dehydrogenase E1 component subunit alpha, mitochondrial & 1.89 & $3.21 \mathrm{E}-02$ \\
\hline \multicolumn{4}{|c|}{ Glycolysis and PPP (7) } \\
\hline TPI1 & Triosephosphate isomerase 1 & 4.21 & 4.88E-05 \\
\hline ALDOA & Fructose-bisphosphate aldolase & 3.60 & 3.45E-04 \\
\hline $\mathrm{PKM} 1 / 2$ & Pyruvate kinase, muscle & 3.26 & $9.79 \mathrm{E}-04$ \\
\hline GAPDH & Glyceraldehyde-3-phosphate dehydrogenase & 2.97 & 2.22E-03 \\
\hline PGAM1 & Phosphoglycerate mutase 1 (Brain) & 2.55 & 6.87E-03 \\
\hline TKT & Transketolase & 2.20 & $1.60 \mathrm{E}-02$ \\
\hline ENO1 & Alpha-enolase & 1.96 & $2.75 \mathrm{E}-02$ \\
\hline \multicolumn{4}{|c|}{ EMT markers and Cytoskeletal proteins (11) } \\
\hline DSP & Desmoplakin & 5.27 & 1.24E-06 \\
\hline FLNB & Filamin-B & 4.81 & $6.21 \mathrm{E}-06$ \\
\hline KRT19 & Keratin, type I cytoskeletal 19 & 4.39 & 2.66E-05 \\
\hline DYNC112 & Cytoplasmic dynein 1 intermediate chain 2 & 3.90 & 1.33E-04 \\
\hline MYL6 & Myosin light polypeptide 6 & 3.74 & $2.22 \mathrm{E}-04$ \\
\hline TUBA1C & Tubulin alpha-1C & 3.30 & 8.64E-04 \\
\hline AGR2 & Anterior gradient protein 2 & 2.94 & $2.44 \mathrm{E}-03$ \\
\hline PAK2 & Serine/threonine-protein kinase PAK 2 & 2.88 & 2.86E-03 \\
\hline TUBB2A & Tubulin beta-2A chain & 2.63 & $5.56 \mathrm{E}-03$ \\
\hline PFN1 & Profilin 1 & 2.27 & 1.36E-02 \\
\hline ARPC2 & Actin related protein $2 / 3$ complex, subunit $2,34 \mathrm{kDa}$, isoform & 2.04 & 2.33E-02 \\
\hline
\end{tabular}

-Transcriptional profiling data derived from the analysis of $\mathrm{N}=28$ breast cancer patients are shown, high-lighting the levels of fold-upregulation observed in the epithelial cancer cell compartment (relative to the tumor stroma), and corresponding $p$-values derived from the analysis of these clinical samples.

To test if mitochondrial inhibitors could block FOXM1-driven mammosphere formation, we then used several independent pharmacological approaches. Interestingly, formation of FOXM1-driven mammospheres was inhibited by treatment with oligomycin A (Figure 8B), XCT790 (Figure 8C), and doxycycline (Figure 8D). Doxycycline is an FDA-approved antibiotic that we have recently shown to inhibit proliferation of TICs, by targeting their mitochondrial ribosomes [12]. Thus, mitochondrial protein translation and function are required for FOXM1-driven mammosphere formation.

\section{Mitochondrial function is required for 3D-spheroid formation using H295R cells}

Finally, we asked if mitochondrial function is required for 3D-spheroid formation of other cell lines, such as H295R adrenocortical carcinoma cells. Figure 9 shows that XCT790 dose-dependently inhibits $3 \mathrm{D}$-spheroid formation of the $\mathrm{H} 295 \mathrm{R}$ adrenocortical carcinoma cell line, with an IC-50 of $10 \mu \mathrm{M}$. These results indicate that mitochondrial function is required for the 3D-spheroid formation of cell lines other than MCF7 breast cancer cells.

Quantitatively similar results were obtained when XCT790 was tested on several other epithelial cancer 
A
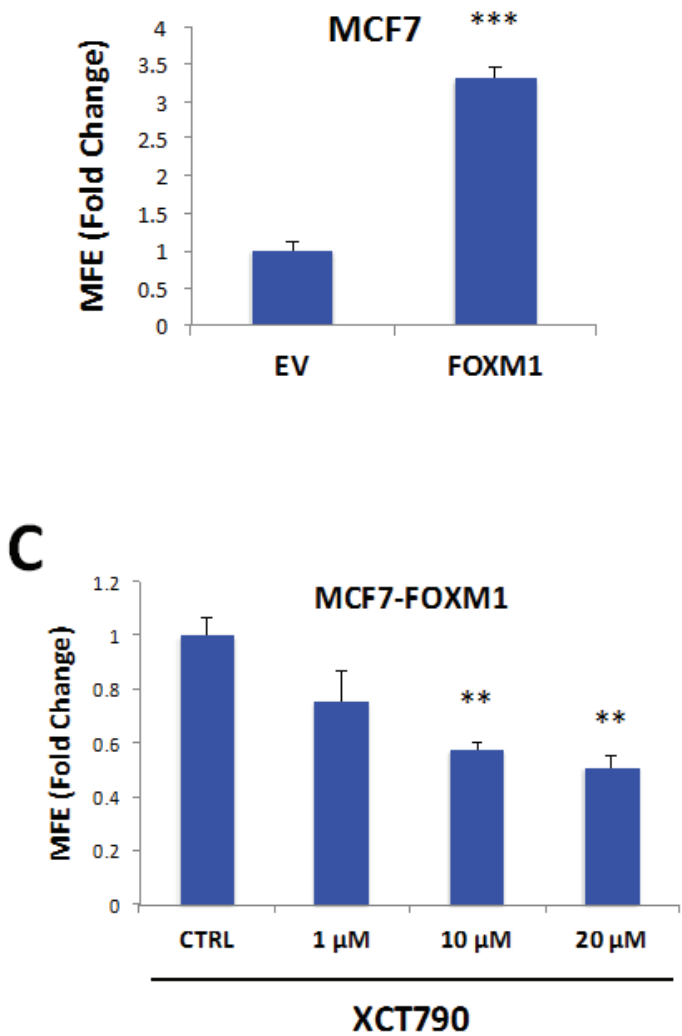

B

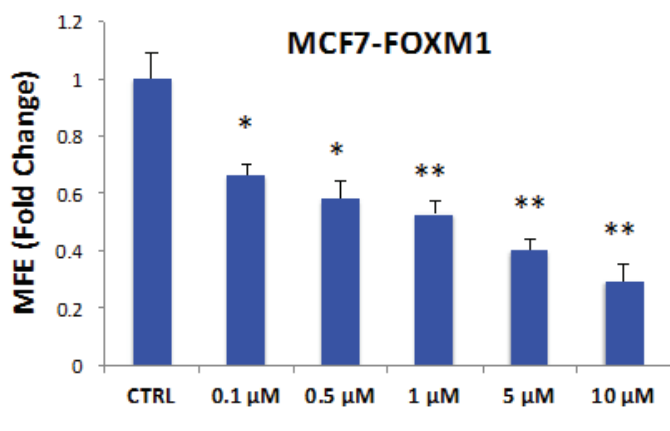

Oligomycin

D

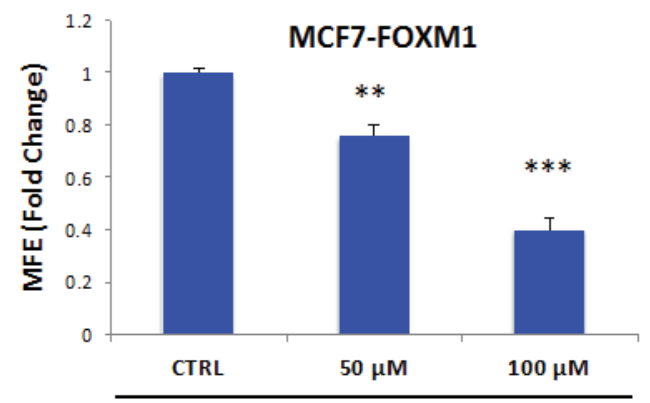

Doxycycline

Figure 8: Mammosphere formation driven by FOXM1 requires mitochondrial function. FOXM1 was over-expressed in MCF7 cells using a lentiviral approach. Empty Vector (EV) cells were generated in parallel. A. MCF7 cells over-expressing FOXM1 show a $>3.3$-fold increase in mammosphere formation. B. Treatment with oligomycin A, an inhibitor of the mitochondrial ATP synthase, inhibits mammosphere formation in MCF7 cells over-expressing FOXM1. C. Treatment with XCT790 inhibits mammosphere formation in MCF7 cells over-expressing FOXM1. D. Treatment with doxycycline, an FDA-approved antibiotic that we have recently shown to inhibit proliferation of TICs by targeting TIC mitochondria, inhibits mammosphere formation in MCF7 cells over-expressing FOXM1. Thus, mitochondrial function is required for FOXM1-driven mammosphere formation. EV: Empty Vector. $* p<0.05,{ }^{* *} p<0.01, * * * p<0.001$ evaluated by Student's $t$ test.

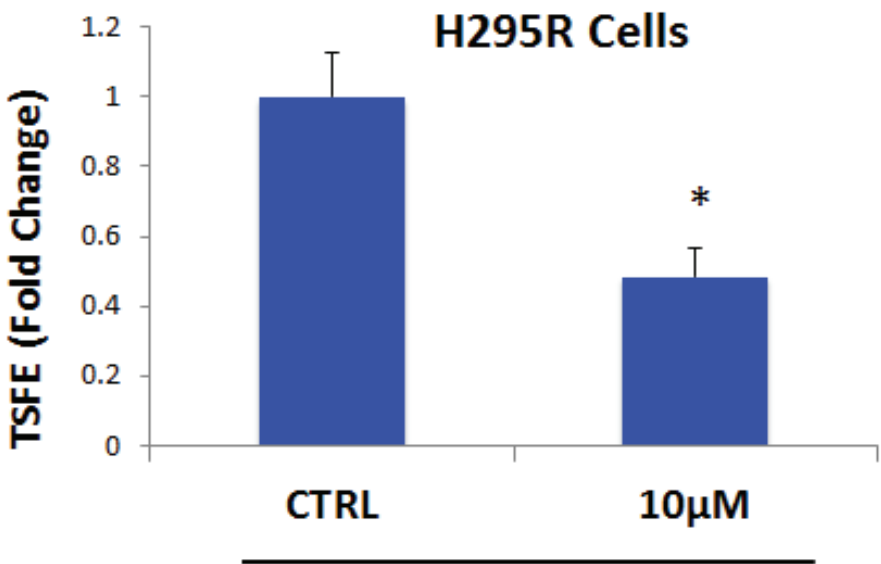

\section{XCT790}

Figure 9: 3D-spheroid formation in H295R cells is dependent upon mitochondrial function. XCT790 dose-dependently inhibits 3D-spheroid formation in H295R cells, an adrenocortical carcinoma cell line, with an IC-50 of $10 \mu \mathrm{M}$. These results indicate that mitochondrial function is required for the 3D-spheroid formation in cell lines other than MCF7 breast cancer cells. TSFE, tumor-sphere forming efficiency; ${ }^{*} p<0.05$ evaluated by Student's $t$ test. 
Table 4: FOXM1 Targets are Transcriptionally Up-regulated in Human Breast Cancer: Ribosomes and Protein Synthesis.

\begin{tabular}{|c|c|c|c|}
\hline Symbol & Gene Description & $\begin{array}{l}\text { Up-regulation } \\
\text { (fold-change) }\end{array}$ & P-value \\
\hline \multicolumn{4}{|c|}{ Ribosome-related proteins (9) } \\
\hline RPL7A & $60 S$ ribosomal protein $L 7 A$ & 5.23 & $1.41 \mathrm{E}-06$ \\
\hline RPL7 & $60 S$ ribosomal protein $\mathrm{L} 7$ & 5.21 & 1.53E-06 \\
\hline RPL10A & $60 S$ ribosomal protein $\mathrm{L} 10 \mathrm{~A}$ & 5.09 & $2.35 \mathrm{E}-06$ \\
\hline MRPL49 & 39S ribosomal protein L49, mitochondrial & 4.94 & $3.93 \mathrm{E}-06$ \\
\hline RPL15 & $60 S$ ribosomal protein $\mathrm{L} 15$ & 4.60 & $1.28 \mathrm{E}-05$ \\
\hline RPS3A & $40 \mathrm{~S}$ ribosomal protein S3A & 4.59 & 1.35E-05 \\
\hline RPL8 & $60 S$ ribosomal protein L8 & 3.86 & $1.51 \mathrm{E}-04$ \\
\hline MRPS22 & $28 \mathrm{~S}$ ribosomal protein S22, mitochondrial & 3.27 & $9.31 \mathrm{E}-04$ \\
\hline RRP1B & Ribosomal RNA processing protein 1 homolog B & 2.21 & $1.58 \mathrm{E}-02$ \\
\hline \multicolumn{4}{|c|}{ Translation initiation factors $(2)$} \\
\hline EIF3C & Eukaryotic translation initiation factor 3 subunit $\mathrm{C}$ & 4.48 & 1.94E-05 \\
\hline EIF4B & Eukaryotic translation initiation factor 4B & 3.17 & 1.27E-03 \\
\hline \multicolumn{4}{|c|}{ Elongation factors (3) } \\
\hline EEF1G & Elongation factor 1-gamma & 3.71 & 2.44E-04 \\
\hline EEF1A1 & Elongation factor 1 -alpha 1 & 3.16 & $1.30 \mathrm{E}-03$ \\
\hline EEF1D & Elongation factor 1-delta & 2.50 & 7.67E-03 \\
\hline \multicolumn{4}{|c|}{ Enzymes for tRNA synthesis (2) } \\
\hline DARS & Aspartate--tRNA ligase, cytoplasmic & 3.43 & $5.87 \mathrm{E}-04$ \\
\hline HARS & Histidine--tRNA ligase, cytoplasmic & 2.42 & $9.55 \mathrm{E}-03$ \\
\hline \multicolumn{4}{|c|}{ Protein folding chaperones (heat shock proteins) (5) } \\
\hline HSP90AB1 & Heat shock protein HSP 90-beta & 4.93 & 5.93E-06 \\
\hline HSPD1 & Heat shock $60 \mathrm{kDa}$ protein 1 (Chaperonin), mitochondrial & 3.42 & 5.93E-04 \\
\hline HSPB1 & Heat shock protein beta- 1 & 3.27 & $9.51 \mathrm{E}-04$ \\
\hline HSPH1 & Heat shock $105 \mathrm{kDa} / 110 \mathrm{kDa}$ protein 1 & 3.18 & $1.22 \mathrm{E}-03$ \\
\hline HSPA8 & Heat shock cognate $71 \mathrm{kDa}$ protein & 2.54 & 7.06E-03 \\
\hline
\end{tabular}

-Transcriptional profiling data derived from the analysis of $\mathrm{N}=28$ breast cancer patients are shown, high-lighting the levels of fold-upregulation observed in the epithelial cancer cell compartment (relative to the tumor stroma), and corresponding $p$-values derived from the analysis of these clinical samples.

cell lines, indicating that the ability of XCT790 to inhibit 3D-spherioid formation reflects a general property of CSCs (data not shown).

\section{Relevance of FOXM1-related targets in human breast cancers}

To assess the possible clinical relevance of our results, we also determined whether the proteomic targets that we identified in MCF7-FOXM1 cells were transcriptionally over-expressed in human breast cancer cells in vivo. Towards this end, we exploited a clinical data set of tumor samples from 28 breast cancer patients. These tumor samples were subjected to laser-capture microdissection, to separate epithelial cancer cells from adjacent tumor stroma [30].

Tables 3 and 4 present a summary of these findings. Overall, greater than fifty FOXM1 targets (related to mitochondria, glycolysis, the EMT, and protein synthesis) that we identified in MCF7-FOXM1 cells were also transcriptionally elevated in human breast cancer cells in vivo. As such, the new FOXM1 protein targets that we identified in MCF7-FOXM1 cells may be especially relevant for improving human breast cancer diagnosis and therapy.

\section{DISCUSSION}

Here, we specifically tested the hypothesis that new mitochondrial biogenesis is required for the survival and propagation of stem-like cancer cells. For this purpose, we used an investigational inhibitor of the ERR $\alpha-\mathrm{PGC} 1 \alpha / \beta$ signaling pathway, namely XCT790, to interrogate the role of mitochondrial biogenesis and function in the anchorageindependent growth of CSCs. Importantly, treatment with XCT790 blocked mammosphere formation and prevented anoikis-resistance in CD44(+)high/CD24(-)low MCF7 cells. Mechanistically, XCT790 inhibited mitochondrial function (OCR) and effectively reduced signaling along a number of classical stem cell transduction pathways, such as the Hedgehog/GLI, TGF $\beta /$ SMAD, and Wnt/ $\beta$ catenin signaling. Overexpression of either ERR $\alpha$ or FOXM1 (a down-stream target of Wnt-signaling [22]) in MCF7 cells significantly augmented mammosphere formation, which could be prevented by a number of mitochondrial inhibitors. In this regard, doxycycline treatment was sufficient to overcome the effects of FOXM1 on mammosphere formation. Interestingly, doxycycline is an FDA-approved antibiotic that has been used for nearly 50 years to treat a wide variety of bacterial and parasitic infections, without significant side effects. Finally, unbiased proteomics analysis of 
MCF7-FOXM1 cells revealed the up-regulation of specific mitochondrial proteins, glycolytic enzymes, EMT markers and components of the protein synthesis machinery. Importantly, these FOXM1 target proteins were also transcriptionally up-regulated in patient samples in vivo, in human breast cancer epithelial cells isolated by laser-capture micro-dissection, highlighting their clinical relevance.

Many other recent studies have also directly implicated augmented mitochondrial function in resistance to chemotherapy and radiation [31-36], in the survival of treatment-resistant cancer stem-like cells [37], in the propagation and motility of circulating tumor cells [38], as well as in tumor growth and cancer cell metastasis in pre-clinical animal models in vivo [39-44].

Consistent with our current findings, we have previously shown that a PGC1/NRF1 gene signature predicts tumor recurrence, metastasis and poor overall survival in $\mathrm{ER}(+) /$ Luminal-A breast cancer patients [45]. This PGC1/NRF1 gene signature was also elevated in $>2,000$ human tumors excised from breast cancer patients, including both ER(+)and ER(-) cases [45]. Moreover, recombinant over-expression of PGC $1 \alpha / \beta$, or other genes that promote mitochondrial biogenesis (MitoNEET/ POLRMT), in MDA-MB-231 cells, was indeed sufficient to functionally increase tumor growth by up to $\sim 3$-fold [43].

Asymmetric cell division is an essential characteristic of stem cells. Interestingly, Sabatini, Weinberg and colleagues examined the segregation of "newly-synthesized" and "older" mitochondria in immortalized mammary epithelial cells, during asymmetric cell division, a property that is also a characteristic of CSCs [46]. They observed that "newlysynthesized" mitochondria were preferentially enriched in stem cells during asymmetric cell division, while "old" mitochondria were segregated into the daughter cells [46]. These results imply that asymmetric cell division in stem cells somehow requires new mitochondrial biogenesis, for the propagation and maintenance of the stem cell phenotype.

As such, these findings may help to mechanistically explain our current results that new mitochondrial biogenesis is required for the efficient propagation of and

\section{CSCs and Mitochondrial Biogenesis}

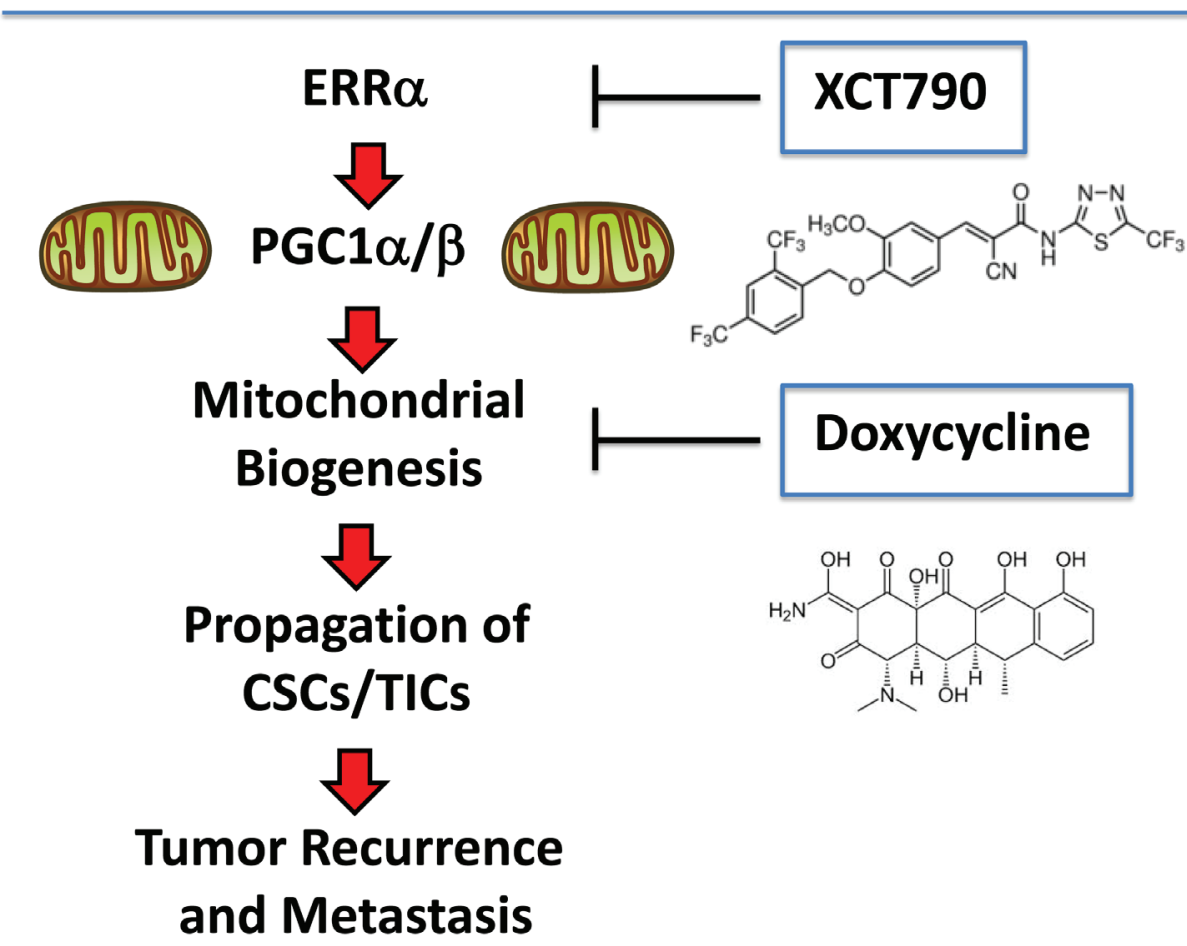

Figure 10: Understanding the role of mitochondrial biogenesis and function in the propagation of CSCs/TICs. ERR $\alpha$ is a co-factor for $\mathrm{PGCl} \alpha / \beta$, which is a well-established transcription factor that is critical for driving new mitochondrial biogenesis. Here, we show that three different classes of mitochondrial inhibitors (XCT790, oligomycin A and doxycycline) all prevent the propagation of CSCs/TICs. XCT790 is an inverse agonist of ERR $\alpha$. Oligomycin A is an inhibitor of the mitochondrial ATP-synthase (complex V) (not shown). Finally, doxycycline is an inhibitor of mitochondrial protein synthesis, as it binds directly to the small subunit of the mitochondrial ribosome. This reflects the fact that mitochondria were originally derived from aerobic bacteria that evolved over millions of years, to establish a symbiotic relationship with the host cell. The chemical structures of XCT790 and doxycycline are also shown. 
survival of CSCs (Figure 10), as we have seen using the mammosphere and anoikis-resistance assays, to measure stem cell activity. Taken together, these data implicate mitochondrial biogenesis and mitochondrial function as critical targets for new drug discovery, to overcome tumor recurrence, distant metastasis and drug-resistance, especially in cancer patients with clinically advanced disease.

\section{MATERIALS AND METHODS}

\section{Materials}

MCF7 breast cancer cells were purchased from the ATCC. H295R adrenocortical carcinoma cells were a generous gift of Dr. Antonio Stigliano (University of Rome). Gibco-brand cell culture media (DMEM/F12) was purchased from Life Technologies. XCT790 was purchased from Tocris, oligomycin A and doxycycline were purchased from Sigma-Aldrich. Lentiviral vectors for the expression of ERR $\alpha(\# Z 1441)$ and FOXM1 (\#U1376) were obtained commercially from Genecopoeia, along with the appropriate empty vector controls, in the Lv-105 (Puro $\left.{ }^{\mathrm{R}}\right)$ vector system.

\section{Mammosphere culture}

A single cell suspension was prepared using enzymatic (1x Trypsin-EDTA, Sigma Aldrich, \#T3924), and manual disaggregation (25 gauge needle) to create a single cell suspension [8]. Cells were plated at a density of 500 cells $/ \mathrm{cm}^{2}$ in mammosphere medium (DMEM-F12/ B27/ EGF(20ng/ml)/ Pen-Strep) in nonadherent conditions, in culture dishes coated with (2-hydroxyethylmethacrylate) (poly-HEMA, Sigma, \#P3932). Cells were grown for 5 days and maintained in a humidified incubator at $37^{\circ} \mathrm{C}$ at an atmospheric pressure in 5\% (v/v) carbon dioxide/air. After 5 days for culture, spheres $>50 \mu \mathrm{m}$ were counted using an eye piece graticule, and the percentage of cells plated which formed spheres was calculated and is referred to as percentage mammosphere formation, and was normalized to one (1 $=100 \% \mathrm{MSE}$, mammosphere forming efficiency). For pharmacological inhibition of mammosphere formation, cells were directly seeded on low-attachment plates in the presence of XCT790 or oligomycin or doxycycline at the indicated concentrations.

\section{Pre-treatment of monolayers with XCT790}

After incubation with XCT790 (5 or $10 \mu \mathrm{M}$ for 48 hours), MCF7 cell monolayers were trypsinized and seeded for mammosphere cultures for 5 days, without
XCT790.

\section{Lentiviruses}

Lentiviral plasmids, packaging cells and reagents were from Genecopoeia. 48 hours after seeding, 293Ta packaging cells were transfected with lentiviral vectors encoding ERR $\alpha$, FOXM1 or empty vector (EX-NEGLv105), using Lenti-PacTM HIV Expression Packaging Kit according to the manufacturer's instructions. Two days post-transfection, lentivirus-containing culture medium was passed through a $0.45 \mu \mathrm{m}$ filter and added to the target cells (MCF7 cells) in the presence of $5 \mu \mathrm{g} / \mathrm{ml}$ Polybrene. Infected cells were selected with a concentration of $1.5 \mu \mathrm{g} /$ $\mathrm{ml}$ of puromycin.

\section{CD44/CD24 analysis}

Following XCT790 treatment for 48 hours, the TIC population was enriched by seeding on low-attachment plates. Under these conditions, the non-TIC population undergoes anoikis (a form of apoptosis induced by a lack of cell-substrate attachment) and TICs are believed to survive. The surviving TIC fraction was analyzed by FACS analysis. Briefly, $1 \times 10^{4} \mathrm{MCF} 7$ monolayer cells were treated with XCT790 $(5 \mu \mathrm{M}$ and $10 \mu \mathrm{M})$ for $48 \mathrm{~h}$ in 6-well plates. Then, cells were trypsinized and seeded in low-attachment plates in mammosphere media. After 10h, MCF7 cells were spun down and incubated with CD24 (IOTest CD24-PE, Beckman Coulter) and CD44 (APC mouse Anti-Human CD44, BD Pharmingen cat.559942) antibodies for 15 minutes on ice. Cells were rinsed twice and incubated with LIVE/DEAD dye (Fixable Dead Violet reactive dye; Invitrogen) for 10 minutes. Samples were then analyzed by FACS (Fortessa, BD Bioscence). Only the live population, as identified by the LIVE/DEAD dye staining, was analyzed for CD24/CD44 expression. Data were analyzed using FlowJo software.

\section{Viability assay}

Cell viability was assessed by sulphorhodamine (SRB) assay, based on the measurement of cellular protein content. After treatment with XCT790 for 3 or 5 days in 96 well plates, cells were fixed with $10 \%$ trichloroacetic acid (TCA) for $1 \mathrm{~h}$ in cold room, and dried overnight at room temperature. Then, cells were incubated with SRB for $15 \mathrm{~min}$, washed twice with $1 \%$ acetic acid, and air dried for at least $1 \mathrm{~h}$. Finally, the protein-bound dye was dissolved in $10 \mathrm{mM}$ Tris $\mathrm{pH} 8.8$ solution and read using the plate reader at $540 \mathrm{~nm}$. 


\section{Evaluation of CSC signalling pathways}

The Cignal Lenti reporter assay (luc) system (Qiagen) was chosen for monitoring the activity of several signaling pathways in MCF7 cells [25]. The responsive luciferase constructs encode the firefly luciferase reporter gene under the control of a minimal (m)CMV promoter and tandem repeats of response elements for each pathway. The following constructs were used: TCF/LEF(luc) for Wnt signaling (CLS-018L); STAT3(luc) for transcriptional activity of STAT3 (CLS-6028L); RBP-Jk(luc) for Notchinduced signaling (CLS-014L); ARE(luc) for Nrf2- and Nrf1-mediated antioxidant response (CLS-2020L); GAS(luc) for IFN $\gamma$-induced Stat1-signaling (CLS-009L); ISRE(luc) for (IFN)- $\alpha / \beta-S T A T 1 / 2$ signaling (CLS-008L); SMAD(luc) for TGF 3 -induced signaling (CLS-017L); GLI(luc) for Sonic hedgehog signaling (CCS-6030L). Briefly, $1 \times 10^{5}$ MCF7 cells were seeded in 12-well plates. Once cells were attached, the viral particles were diluted $1: 10$ in complete culture media containing polybrene (sc134220, Santa Cruz), and added to the cells. Puromycin treatment (\#P9620, Sigma) was started 48 hours later in order to select stably infected cells.

\section{Luciferase assay}

Luciferase Assay System (E1501, Promega) was performed in all luciferase reporter MCF7 cells treated with XCT790. Briefly, $6 \times 10^{3}$ MCF7 cells were seeded in black-walled 96-well plates and then were treated with XCT790 $(10 \mu \mathrm{g} / \mathrm{ml})$. As control, vehicle-treated cells were run in parallel. Four replicates were used for each condition. After 48 hours of treatment, luciferase assay was performed according to the manufacturer's instructions. Light signal was acquired for 2 minutes in photons/second in the Xenogen VivoVision IVIS Lumina (Caliper Life Sciences), and the results were analysed using the Living Image 3.2 sofware (Caliper Life Sciences). Luminescence was normalized using total proteins, as assessed with the Bradford protein assay.

\section{Mitochondrial staining}

To measure mitochondrial activity, cells were stained with MitoTracker Orange (\#M7510, Invitrogen), whose accumulation in mitochondria is dependent upon membrane potential. To measure mitochondrial mass, cells were stained with MitoTracker Green (\#M7514 Invitrogen), or MitoTracker Deep Red (\#M22426, Invitrogen), both localizing to mitochondria regardless of mitochondrial membrane potential. Briefly, MCF7 cells were treated with XCT790 for 48 hours. Cells were then incubated with pre-warmed MitoTracker staining solution (diluted in PBS/CM to a final concentration of $10 \mathrm{nM}$ ) for
30-60 min at $37{ }^{\circ} \mathrm{C}$. All subsequent steps were performed in the dark. Cells were washed in PBS, harvested, and resuspended in $300 \mu \mathrm{L}$ of PBS. Cells were then analyzed by flow cytometry. Data analysis was performed using FlowJo software.

\section{Seahorse XFe96 metabolic flux analysis}

Extracellular acidification rates (ECAR) and realtime oxygen consumption rates (OCR) for MCF7 cells treated with XCT790 or vehicle alone control were determined using the Seahorse Extracellular Flux (XF96) analyzer (Seahorse Bioscience, MA, USA). MCF7 cells were maintained in DMEM supplemented with $10 \%$ FBS (fetal bovine serum), $2 \mathrm{mM}$ GlutaMAX, and 1\% PenStrep. 7,000 cells were seeded per well into XF96-well cell culture plates, and incubated overnight at $37^{\circ} \mathrm{C}$ in a $5 \% \mathrm{CO}_{2}$ humidified atmosphere. After $24 \mathrm{~h}$, cells were treated with XCT790 $(5 \mu \mathrm{M}$ or $10 \mu \mathrm{M})$ for $48 \mathrm{~h}$. After $48 \mathrm{~h}$ of treatment, cells were washed in pre-warmed XF assay media (for OCR measurement, XF assay media was supplemented with $10 \mathrm{mM}$ glucose, $1 \mathrm{mM}$ Pyruvate, $2 \mathrm{mM} \mathrm{L}$-glutamine and adjusted at $\mathrm{pH}$ 7.4). Cells were then maintained in $175 \mu \mathrm{L} /$ well of XF assay media at $37^{\circ} \mathrm{C}$, in a non- $\mathrm{CO}_{2}$ incubator for $1 \mathrm{~h}$. During the cell incubation time, we loaded $25 \mu \mathrm{L}$ each of $80 \mathrm{mM}$ glucose, $9 \mu \mathrm{M}$ oligomycin, 1M 2-deoxyglucose (for ECAR measurement) and $25 \mu \mathrm{L}$ each of $10 \mu \mathrm{M}$ oligomycin, $9 \mu \mathrm{M}$ FCCP, $10 \mu \mathrm{M}$ rotenone, $10 \mu \mathrm{M}$ antimycin A (for OCR measurement), in XF assay media into the injection ports in the XFe-96 sensor cartridge. ECAR and OCR measurements were normalized by protein content. Data set was analyzed by XFe-96 software and GraphPad Prism software, using one-way ANOVA and Student's t-test calculations. All experiments were performed in quintuplicate, three times independently, such that each data point represents the average of 15 replicates.

\section{Label-free quantitative proteomics analysis}

Cell lysates were prepared for trypsin digestion by sequential reduction of disulphide bonds with TCEP and alkylation with MMTS [47]. Then, the peptides were extracted and prepared for LC-MS/MS. All LC-MS/MS analyses were performed on an LTQ Orbitrap XL mass spectrometer (Thermo Scientific, San Jose, CA) coupled to an Ultimate 3000 RSLCnano system (Thermo Scientific, formerly Dionex, The Netherlands). Xcalibur raw data files acquired on the LTQ-Orbitrap XL were directly imported into Progenesis LCMS software (Waters Corp., Milford, MA, formerly Non-linear dynamics, Newcastle upon Tyne, UK) for peak detection and alignment. Data were analyzed using the Mascot search engine. Five replicates were analyzed for each sample type $(N=5)$. Statistical analyses were performed using ANOVA and 
only fold-changes in proteins with a p-value less than 0.05 were considered significant.

\section{Data mining}

To firmly establish the clinical relevance of our results from the quantitative proteomics analysis of mammosheres, we re-analyzed the transcriptional profiles of epithelial breast cancer cells and adjacent tumor stromal cells that were physically separated by lasercapture microdissection (from $N=28$ human breast cancer patients) [30].

\section{ACKNOWLEDGMENTS}

We thank the University of Manchester for providing start-up funds that contributed to the success of this study. FS and MPL were supported, in part, by funding from the European Union (ERC Advanced Grant), Breakthrough Breast Cancer, and the Manchester Cancer Research Centre (MCRC). DLS was core-funded by CRUK. ADL was supported by an E.U. grant for post-doctoral associates: Unical (POR Calabria FSE 2007-2013. VP was funded by a grant from the Associazione Italiana per la Ricerca sul Cancro (AIRC), project n. IG14433.

\section{CONFLICTS OF INTEREST}

There is no conflict of interest.

\section{REFERENCES}

1. Sinha N, Mukhopadhyay S, Das DN, Panda PK and Bhutia SK. Relevance of cancer initiating/stem cells in carcinogenesis and therapy resistance in oral cancer. Oral oncology. 2013; 49:854-862.

2. Scopelliti A, Cammareri P, Catalano V, Saladino V, Todaro M and Stassi G. Therapeutic implications of Cancer Initiating Cells. Expert opinion on biological therapy. 2009; 9:1005-1016.

3. Xin H, Kong Y, Jiang X, Wang K, Qin X, Miao ZH, Zhu $\mathrm{Y}$ and Tan W. Multi-drug-resistant cells enriched from chronic myeloid leukemia cells by Doxorubicin possess tumor-initiating-cell properties. Journal of pharmacological sciences. 2013; 122:299-304.

4. Easwaran H, Tsai HC and Baylin SB. Cancer epigenetics: tumor heterogeneity, plasticity of stem-like states, and drug resistance. Molecular cell. 2014; 54:716-727.

5. Takebe N, Miele L, Harris PJ, Jeong W, Bando H, Kahn M, Yang SX and Ivy SP. Targeting Notch, Hedgehog, and Wnt pathways in cancer stem cells: clinical update. Nature reviews Clinical oncology. 2015.

6. Angeloni V, Tiberio P, Appierto V and Daidone MG. Implications of stemness-related signaling pathways in breast cancer response to therapy. Seminars in cancer biology. 2015; 31:43-51.

7. Maccalli C and De Maria R. Cancer stem cells: perspectives for therapeutic targeting. Cancer immunology, immunotherapy : CII. 2015; 64:91-97.

8. Shaw FL, Harrison H, Spence K, Ablett MP, Simoes BM, Farnie $\mathrm{G}$ and Clarke RB. A detailed mammosphere assay protocol for the quantification of breast stem cell activity. Journal of mammary gland biology and neoplasia. 2012; 17:111-117.

9. Alvero AB, Montagna MK, Holmberg JC, Craveiro V, Brown D and Mor G. Targeting the mitochondria activates two independent cell death pathways in ovarian cancer stem cells. Molecular cancer therapeutics. 2011; 10:1385-1393.

10. Pasto A, Bellio C, Pilotto G, Ciminale V, Silic-Benussi M, Guzzo G, Rasola A, Frasson C, Nardo G, Zulato E, Nicoletto MO, Manicone M, Indraccolo S and Amadori A. Cancer stem cells from epithelial ovarian cancer patients privilege oxidative phosphorylation, and resist glucose deprivation. Oncotarget. 2014; 5:4305-4319.

11. Lamb R, Harrison H, Hulit J, Smith DL, Lisanti MP and Sotgia F. Mitochondria as new therapeutic targets for eradicating cancer stem cells: Quantitative proteomics and functional validation via MCT1/2 inhibition. Oncotarget. 2014; 5:11029-11037.

12. Lamb R, Ozsvari B, Lisanti CL, Tanowitz HB, Howell A, Martinez-Outschoorn UE, Sotgia F and Lisanti MP. Antibiotics that target mitochondria effectively eradicate cancer stem cells, across multiple tumor types: Treating cancer like an infectious disease. Oncotarget. 6:4569-4584.

13. Vlashi E, Lagadec C, Vergnes L, Matsutani T, Masui K, Poulou M, Popescu R, Della Donna L, Evers P, Dekmezian C, Reue K, Christofk H, Mischel PS and Pajonk F. Metabolic state of glioma stem cells and nontumorigenic cells. Proceedings of the National Academy of Sciences of the United States of America. 2011; 108:16062-16067.

14. Vlashi E, Lagadec C, Vergnes L, Reue K, Frohnen P, Chan M, Alhiyari Y, Dratver MB and Pajonk F. Metabolic differences in breast cancer stem cells and differentiated progeny. Breast cancer research and treatment. 2014; 146:525-534.

15. Busch BB, Stevens WC, Jr., Martin R, Ordentlich P, Zhou S, Sapp DW, Horlick RA and Mohan R. Identification of a selective inverse agonist for the orphan nuclear receptor estrogen-related receptor alpha. Journal of medicinal chemistry. 2004; 47:5593-5596.

16. Deblois $\mathrm{G}$ and Giguere V. Functional and physiological genomics of estrogen-related receptors (ERRs) in health and disease. Biochimica et biophysica acta. 2011; 1812:10321040 .

17. Deblois G, St-Pierre J and Giguere V. The PGC-1/ERR signaling axis in cancer. Oncogene. 2013; 32:3483-3490.

18. Fisher KW, Das B, Kortum RL, Chaika OV and Lewis RE. Kinase suppressor of ras 1 (KSR1) regulates PGC1alpha 
and estrogen-related receptor alpha to promote oncogenic Ras-dependent anchorage-independent growth. Molecular and cellular biology. 2011; 31:2453-2461.

19. Wu F, Wang J, Wang Y, Kwok TT, Kong SK and Wong C. Estrogen-related receptor alpha (ERRalpha) inverse agonist XCT-790 induces cell death in chemotherapeutic resistant cancer cells. Chemico-biological interactions. 2009; 181:236-242.

20. Cassano P, Sciancalepore AG, Pesce V, Fluck M, Hoppeler $\mathrm{H}$, Calvani M, Mosconi L, Cantatore P and Gadaleta MN. Acetyl-L-carnitine feeding to unloaded rats triggers in soleus muscle the coordinated expression of genes involved in mitochondrial biogenesis. Biochimica et biophysica acta. 2006; 1757:1421-1428.

21. Gemenetzidis E, Elena-Costea D, Parkinson EK, Waseem A, Wan H and Teh MT. Induction of human epithelial stem/ progenitor expansion by FOXM1. Cancer research. 2010; 70:9515-9526.

22. Zhang N, Wei P, Gong A, Chiu WT, Lee HT, Colman H, Huang H, Xue J, Liu M, Wang Y, Sawaya R, Xie K, Yung WK, Medema RH, He X and Huang S. FoxM1 promotes beta-catenin nuclear localization and controls Wnt targetgene expression and glioma tumorigenesis. Cancer cell. 2011; 20:427-442.

23. Bao B, Wang Z, Ali S, Kong D, Banerjee S, Ahmad A, Li Y, Azmi AS, Miele L and Sarkar FH. Over-expression of FoxM1 leads to epithelial-mesenchymal transition and cancer stem cell phenotype in pancreatic cancer cells. Journal of cellular biochemistry. 2011; 112:2296-2306.

24. Chiu WT, Huang YF, Tsai HY, Chen $\mathrm{CC}$, Chang $\mathrm{CH}$, Huang SC, Hsu KF and Chou CY. FOXM1 confers to epithelial-mesenchymal transition, stemness and chemoresistance in epithelial ovarian carcinoma cells. Oncotarget. 2015; 6:2349-2365.

25. Peiris-Pages M, Sotgia F and Lisanti MP. Chemotherapy induces the cancer-associated fibroblast phenotype, activating paracrine hedgehog-GLI signaling in breast cancer cells. Oncotarget. 6:10728-10745.

26. Bergamaschi A, Christensen BL and Katzenellenbogen BS. Reversal of endocrine resistance in breast cancer: interrelationships among 14-3-3zeta, FOXM1, and a gene signature associated with mitosis. Breast cancer research : BCR. 2011; 13:R70.

27. Lamb R, Harrison H, Smith DL, Townsend PA, Jackson T, Ozsvari B, Martinez-Outschoorn UE, Pestell RG, Howell A, Lisanti MP and Sotgia F. Targeting tumor-initiating cells: eliminating anabolic cancer stem cells with inhibitors of protein synthesis or by mimicking caloric restriction. Oncotarget. 2015; 6:4585-4601.

28. Scheel C, Eaton EN, Li SH, Chaffer CL, Reinhardt F, Kah KJ, Bell G, Guo W, Rubin J, Richardson AL and Weinberg RA. Paracrine and autocrine signals induce and maintain mesenchymal and stem cell states in the breast. Cell. 2011; 145:926-940.
29. Mani SA, Guo W, Liao MJ, Eaton EN, Ayyanan A, Zhou AY, Brooks M, Reinhard F, Zhang CC, Shipitsin M, Campbell LL, Polyak K, Brisken C, Yang J and Weinberg RA. The epithelial-mesenchymal transition generates cells with properties of stem cells. Cell. 2008; 133:704-715.

30. Casey T, Bond J, Tighe S, Hunter T, Lintault L, Patel O, Eneman J, Crocker A, White J, Tessitore J, Stanley M, Harlow S, Weaver D, Muss H and Plaut K. Molecular signatures suggest a major role for stromal cells in development of invasive breast cancer. Breast cancer research and treatment. 2009; 114:47-62.

31. Bowling BD, Doudican N, Manga P and Orlow SJ. Inhibition of mitochondrial protein translation sensitizes melanoma cells to arsenic trioxide cytotoxicity via a reactive oxygen species dependent mechanism. Cancer chemotherapy and pharmacology. 2008; 63:37-43.

32. Martinez-Outschoorn UE, Goldberg A, Lin Z, Ko YH, Flomenberg N, Wang C, Pavlides S, Pestell RG, Howell A, Sotgia F and Lisanti MP. Anti-estrogen resistance in breast cancer is induced by the tumor microenvironment and can be overcome by inhibiting mitochondrial function in epithelial cancer cells. Cancer biology \& therapy. 2011; 12:924-938.

33. Martinez-Outschoorn UE, Prisco M, Ertel A, Tsirigos A, Lin Z, Pavlides S, Wang C, Flomenberg N, Knudsen ES, Howell A, Pestell RG, Sotgia F and Lisanti MP. Ketones and lactate increase cancer cell "stemness," driving recurrence, metastasis and poor clinical outcome in breast cancer: achieving personalized medicine via MetaboloGenomics. Cell cycle. 2011; 10:1271-1286.

34. Lu CL, Qin L, Liu HC, Candas D, Fan M and Li JJ. Tumor cells switch to mitochondrial oxidative phosphorylation under radiation via mTOR-mediated hexokinase II inhibition--a Warburg-reversing effect. PloS one. 2015; 10:e0121046.

35. Whitaker-Menezes D, Martinez-Outschoorn UE, Flomenberg N, Birbe RC, Witkiewicz AK, Howell A, Pavlides S, Tsirigos A, Ertel A, Pestell RG, Broda P, Minetti C, Lisanti MP and Sotgia F. Hyperactivation of oxidative mitochondrial metabolism in epithelial cancer cells in situ: visualizing the therapeutic effects of metformin in tumor tissue. Cell cycle. 2011; 10:4047-4064.

36. Ko YH, Lin Z, Flomenberg N, Pestell RG, Howell A, Sotgia F, Lisanti MP and Martinez-Outschoorn UE. Glutamine fuels a vicious cycle of autophagy in the tumor stroma and oxidative mitochondrial metabolism in epithelial cancer cells: implications for preventing chemotherapy resistance. Cancer biology \& therapy. 2011; 12:1085-1097.

37. Viale A, Pettazzoni P, Lyssiotis CA, Ying H, Sanchez N, Marchesini M, Carugo A, Green T, Seth S, Giuliani V, Kost-Alimova M, Muller F, Colla S, Nezi L, Genovese G, Deem AK, et al. Oncogene ablation-resistant pancreatic cancer cells depend on mitochondrial function. Nature. 2014; 514:628-632.

38. LeBleu VS, O’Connell JT, Gonzalez Herrera KN, Wikman 
H, Pantel K, Haigis MC, de Carvalho FM, Damascena A, Domingos Chinen LT, Rocha RM, Asara JM and Kalluri R. PGC-1alpha mediates mitochondrial biogenesis and oxidative phosphorylation in cancer cells to promote metastasis. Nature cell biology. 2014; 16:992-1003, 10011015.

39. Bonuccelli G, Tsirigos A, Whitaker-Menezes D, Pavlides S, Pestell RG, Chiavarina B, Frank PG, Flomenberg N, Howell A, Martinez-Outschoorn UE, Sotgia F and Lisanti MP. Ketones and lactate "fuel" tumor growth and metastasis: Evidence that epithelial cancer cells use oxidative mitochondrial metabolism. Cell cycle. 2010; 9:3506-3514.

40. Martinez-Outschoorn UE, Sotgia F and Lisanti MP. Power surge: supporting cells "fuel" cancer cell mitochondria. Cell metabolism. 2012; 15:4-5.

41. Son J, Lyssiotis CA, Ying H, Wang X, Hua S, Ligorio M, Perera RM, Ferrone CR, Mullarky E, Shyh-Chang N, Kang Y, Fleming JB, Bardeesy N, Asara JM, Haigis MC, DePinho RA, et al. Glutamine supports pancreatic cancer growth through a KRAS-regulated metabolic pathway. Nature. 2013; 496:101-105.

42. Tan AS, Baty JW, Dong LF, Bezawork-Geleta A, Endaya B, Goodwin J, Bajzikova M, Kovarova J, Peterka M, Yan B, Pesdar EA, Sobol M, Filimonenko A, Stuart S, Vondrusova M, Kluckova K, et al. Mitochondrial genome acquisition restores respiratory function and tumorigenic potential of cancer cells without mitochondrial DNA. Cell metabolism. 2015; 21:81-94.

43. Salem AF, Whitaker-Menezes D, Howell A, Sotgia F and Lisanti MP. Mitochondrial biogenesis in epithelial cancer cells promotes breast cancer tumor growth and confers autophagy resistance. Cell cycle. 2012; 11:4174-4180.

44. Martinez-Outschoorn UE, Sotgia F and Lisanti MP. Caveolae and signalling in cancer. Nature reviews Cancer. 2015; 15:225-237.

45. Ertel A, Tsirigos A, Whitaker-Menezes D, Birbe RC, Pavlides S, Martinez-Outschoorn UE, Pestell RG, Howell A, Sotgia F and Lisanti MP. Is cancer a metabolic rebellion against host aging? In the quest for immortality, tumor cells try to save themselves by boosting mitochondrial metabolism. Cell Cycle. 2012; 11:253-63.

46. Katajisto P, Dohla J, Chaffer CL, Pentinmikko N, Marjanovic N, Iqbal S, Zoncu R, Chen W, Weinberg RA and Sabatini DM. Stem cells. Asymmetric apportioning of aged mitochondria between daughter cells is required for stemness. Science. 2015; 348:340-343.

47. Holland M, Castro FV, Alexander S, Smith D, Liu J, Walker M, Bitton D, Mulryan K, Ashton G, Blaylock M, Bagley S, Connolly Y, Bridgeman J, Miller C, Krishnan S, Dempsey $\mathrm{C}$, et al. RAC2, AEP, and ICAM1 expression are associated with CNS disease in a mouse model of pre-B childhood acute lymphoblastic leukemia. Blood. 2011; 118:638-649. 